ANL -6512

Reactor Technology

(TID-4500, 17th Ed.)

AEC Research and

Development Report

ARGONNE NATIONAL LABORATORY

9700 South Cass Avenue

Argonne, Illinois

A THEORETICAL STUDY OF

DESTRUCTIVE NUCLEAR BURSTS

IN EAST POWER REACTORS

by

V.Z. Jankus

Reactor Engineering Division

February 1962

Operated by The University of Chicago

under

Contract $W-31-109-$ eng-38 


\section{DISCLAIMER}

This report was prepared as an account of work sponsored by an agency of the United States Government. Neither the United States Government nor any agency Thereof, nor any of their employees, makes any warranty, express or implied, or assumes any legal liability or responsibility for the accuracy, completeness, or usefulness of any information, apparatus, product, or process disclosed, or represents that its use would not infringe privately owned rights. Reference herein to any specific commercial product, process, or service by trade name, trademark, manufacturer, or otherwise does not necessarily constitute or imply its endorsement, recommendation, or favoring by the United States Government or any agency thereof. The views and opinions of authors expressed herein do not necessarily state or reflect those of the United States Government or any agency thereof. 


\section{DISCLAIMER}

Portions of this document may be illegible in electronic image products. Images are produced from the best available original document. 
TABLE OF CONTENTS

Page

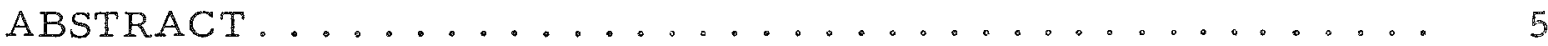

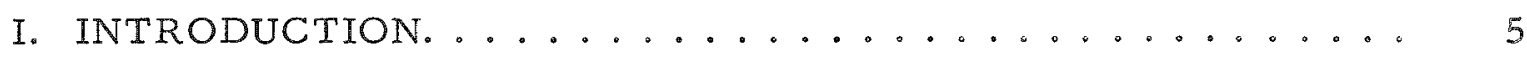

II. THE BETHE-TAIT METHOD ............... 7

A. Mechanism of Reactivity Reduction ............ 7

B. Solution of Kinetic Equations............... 12

C. Excess Energy and an Upper Bound for Mechanical

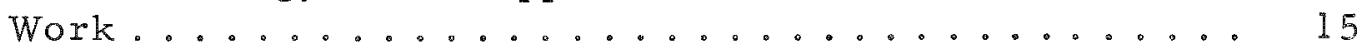

D. Approximate Solution of Kinetic Equations ......... 17

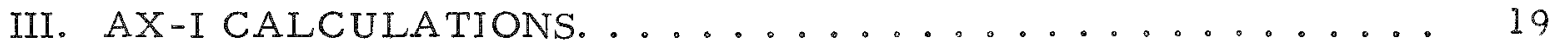

A. General Description of the Code.............. 20

B. Common Procedure Used in Present Study .......... 21

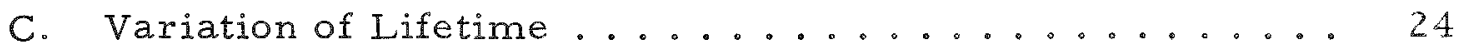

D. Variation of Blanket Density............... 26

E. Yield as a Function of $k_{0}$ and $k_{0}^{3} / k^{2} \ldots \ldots \ldots 27$

F. Variation of Wave Velocity................ 29

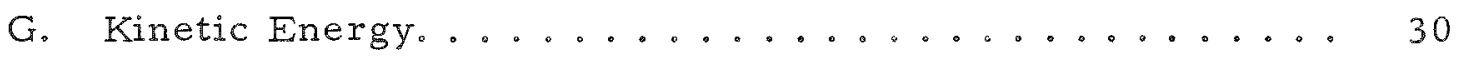

Appendix A - ON THE EQUATIONS OF STATE USED IN AX-I ... 33

Appendix B - CONNECTION BETWEEN NUCLEAR PARAMETERS $k_{0}, \ell, A N D$ THE EQUATION OF STATE ....... 38

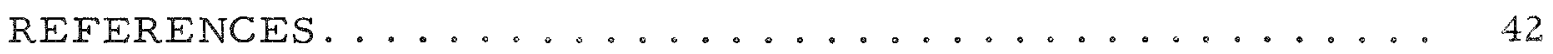

ACKNOWLEDGMENTS ........................ 43 


\section{LIST OF FIGURES}

No.

Title

Page

1. Ratio of Excess Energy to Threshold Energy in Simple Bethe-Tait Calculation ................ 44

2. Width of the Power Burst in Terms of Initial Period..... 44

3. An Upper Bound for Maximum Work as Given by Eq. (21) . . 45

4. Ratio of Excess Energy in BT to that in Approximation BT ${ }^{\prime}$. 45

5. Ratio of Excess Energy in $\mathrm{AX}-\mathrm{I}$ to that in $\mathrm{AX}-\mathrm{I}^{\prime}$ : $\mathrm{x} \approx 1.10 \times 10^{4}\left(k_{0}^{3} / \ell^{2}\right) \ldots \ldots \ldots \ldots$

6. Energy Yield in Excess of Threshold for Various Life-

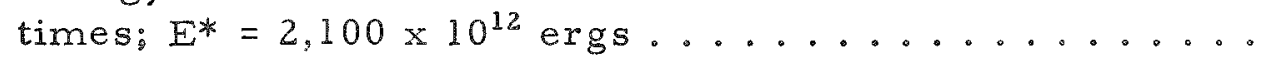

7. Center Peak Pressure for Various Lifetimes: $(\partial \mathrm{p} / \partial \mathrm{e})_{\rho} \mathrm{Q}^{*}=0.126$ megabar $\ldots \ldots . \ldots . \ldots 46$

8. Effect of Blanket Density............... 47

9. Variation of Center Peak Pressure with Wave Velocity... 47

10. Dimensionless Excess Energy Yield, $E_{e x} / E^{*}$, as a Function of Dimensionless Parameters $x \mathrm{Eq} .(32)$ and y Eq. (6b): $\tau^{\prime} /\left(\tau^{\prime}+\beta^{\prime} \theta_{i}\right)=1.0776 \ldots \ldots \ldots \ldots$

11. Dimensionless Center Peak Pressure, Pmax $/\left(-\tau^{\prime}-\beta^{\prime} \theta_{i}\right)$, as a Function of Dimensionless Parameters $x$ Eq. (32) and $y$ Eq. $(6 \mathrm{~b}): \tau^{\prime} /\left(\tau^{1}+\beta^{1} \theta_{\mathrm{i}}\right)=1.0776 \ldots \ldots \ldots$ 
Page

I. Ratio of Excess Energy to Threshold Energy, $\left(Q-Q^{*}\right) / Q^{*}$, in Simple Bethe-Tait Calculation.............

II. An Upper Bound for Maximum Work, $3 q^{3 / 2} W_{\max } / 4 \pi b^{3} \rho_{c} Q^{*}$,

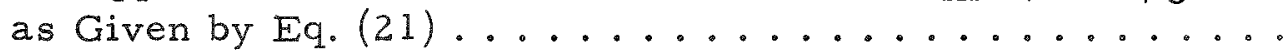

III. The Peak of the (Dimensionless) Average Core Pressure, Eqs. (22) or (8b), if Pressure is Proportional to $n^{\text {th }}$ Power

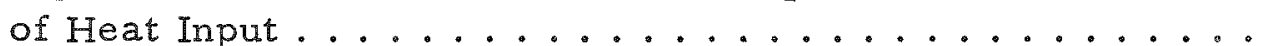

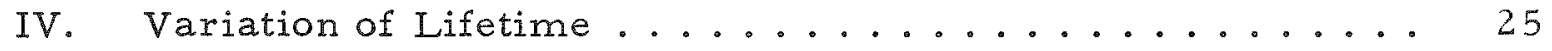

V. Variation of Blanket Density.............. 27

VI. Variation of Reactivity Input................ 29

VII. Variation of Wave Velocity................. 30

VIII. Linearization of Pressure Dependence on Density ..... 36

IX. Connection Between Nuclear and Equation of State

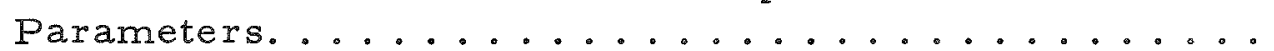




\title{
A THEORETICAL STUDY OF \\ DESTRUCTIVE NUCLEAR BURSTS \\ IN FAST POWER REACTORS
}

by

V. Z. Jankus

\begin{abstract}
This report describes the calculation of destructive nuclear bursts in fast reactors by an improved Bethe-Tait method, which, for purposes of calculation, neglects propagation of the pressure wave. Then exact numerical calculations for hydrodynamic and neutronic conditions during the power burst are performed in order to assors the importance of this neglect.
\end{abstract}

\section{INTRODUCTION}

In this study we will consider large, uncontrolled power bursts in fast reactors. We will assume that an appreciable amount of reactivity is somehow inserted into the reactor, power keeps on rising, and heat is carried away until eventually in the reactor occur changes that lead to reduction of reactivity and the shutdown of power generation.

Concurrent with increase in temperature, effective microscopic cross sections may change. In a thermal reactor, change of diffusion length may be a major factor leading towards reactivity reduction. In a fast reactor, thermal neutrons are unimportant, but effective microscopic cross sections may be influenced by Doppler broadening in the region of resonances. The Doppler effect is small for metal-fueled cores and, for the sake of simplicity, will be neglected in present study. Besides changes of microscopic cross sections, increase of temperature causes increases in pressure, motion of reactor materials, and, connected with it, changes of density. If the excursion is very slow, heat produced in the core is conducted to the blanket and supporting structure, and the reactor is shit $7 . \cdots \mathrm{m}$ by an expansion of the whole structure. For a faster excursion, the rate of heat conduction lags appreciably behind the rate of heat production. In a thermal reactor especially, conduction of heat from fuel elements to the moderator and expulsion of the moderator may be of major significance. For sharp excursions considered in this report we neglect heat conduction entirely. Time scales of fast reactor bursts are so short, of the order of a few hundred microseconds, that no significant heat conduction is possible. 
We neglect also rigidity of reactor structure and assume that pressures generated by heating reactor material are relieved by its motion, possibly transferred along to the unheated portions by the propagation of the pres sure wave. Delayed neutrons are also neglected. And only excursions starting with step insertion of prompt reactivity $k_{0}$ are treated explicitly. However, this describes also the behavior of large excursions caused by ramp insertion of reactivity, during the short time reactivity reduction mechanism is operative. (2)

If an excursion is so sharp that even the pressure wave does not have time to propagate an appreciable distance, the reactivity reduction is impeded principally by the inertia of mass itself. Such a case is amenable to a semi-analytical treatment, performed first in a paper by Bethe and Tait. (1) In the following chapter we will consider the application of their method for a general case, and we will see that the yields depend on several functions describing the reactor. Usually, however, the peak of average pressure multiplied by $l^{2} / k_{0}^{3}$ (where $l$ is the prompt lifetime) is not a sensitive function of reactivity input $k_{0}$.

The next chapter describes a number of calculations that treat reactor neutronics and hydrodynamics more exactly. The calculations have been performed by means of a complicated numerical code, AX-I. ( ) They show that wave propagation is of lesser importance for determination of energy yield than for determination of actual pressures and displacement of reactor material. The salient outcome in all the cases considered is that the Bethe-Tait method tends to predict, for the same accident, a lower integrated energy yield and a higher pressure than the more accurate $A X-I$ calculation. Also, the Bethe-Tait results get closer to the results of AX-I as the magnitude of the excursion increases. Thus one can use the BetheTait method to give a pessimistic estimate of pressure peak and an optimistic estimate of energy yield. The estimates are close to the actual situation, of course, only for very large excursions. 


\section{THE BETHE-TAIT METHOD}

An analytical method of calculating the energy release in large nuclear bursts in fast reactors was given by Bethe and Tart (1) In this calculation they have assumed that the power keeps rising exponentially. From the resulting energy generated, they have calculated pressures, neglecting local expansion. Then, using pressures obtained this way, and neglecting propagation, they have obtained displacements and reactivity reduction. The calculation is ended when prompt reactivity was reduced to zero. The use of a constant rate of exponential power rise during the positive period range and the neglect of power generated thereafter have somewhat compensating tendencies. The exact consequences of this assumption, however, called for some investigation. Thus, an expression for reactivity reduction has been obtained for arbitrary variation of power with time. (2) In this derivation, the intermediate steps in Bethe-Tait procedure have been telescoped analytically and an expression for second derivative of reactivity as function of energy input has been derived. In the original Bethe-Tait paper only spherically symmetric geometry was used, the shape of power density was assumed to be parabolic, and only an equation of state with threshold for power generation has been used. Also, the excursion considered in the original paper was of low yield, and the expansion of pressure into the blanket did not have to be considered. Here we allow also for expansion of pressure into the blanket and demonstrate also that other as sumptions are also nonessential for the treatment of this type.

In Section II-A we derive a completely general formula which assumes only perturbation treatment of reactivity and for evaluation of which we need only to neglect wave propagation and keep power density of the same spatial shape.

In Section II-B we evaluate our expression using all the original Bethe-Tait assumptions, but make the variation of power with time arbitrary. Then, using a simple numerical procedure, we determine variation of power with time and total energy yield.

In Section II-D we compare these results with results obtained by the original Bethe-Tait method and show that original method underestimates the yield substantially for smaller excursions.

In Section II-C it is recognized that the total energy yield is not a good indication of destructive potential if the equation of state has a large threshold for pressure generation and a smaller quantity is derived.

A. Mechanism of Reactivity Reduction

In a previous paper, (2) an expression for the reactivity reduction was derived based on a first-order perturbation of the one-group diffusion 
equation. The somewhat doubtful accuracy of the one-group treatment might be relieved by generalizing the treatment to the use of a number of groups. Numerical calculation in such cases, however, is rather cumbersome. Thus, here we intend to derive equivalent expressions based on the (experimentally measurable) worth of reactor material. R. D. Nicholson(4) has applied this idea in a study of quasi-two-dimensional explosion calculations. Also, we wish to extend the calculation (consistent with the BetheTait assumptions) for the case in which the "microscopic" transport cross section in the blanket is different from that in the core.

We will consider a reactor composed of a core and a blanket. We will assume that the core is composed of one homogenized material, the blanket of another, and that there may be variation of density of these materials. We will define the worth of reactor material, $w(r)$, as the decrease in reactivity due to removal of unit mass of material at the position $r$. The worth will be continuous in the reactor except for the interface of the core and the blanket. The change in the reactivity due to an increase in density, $\delta \rho(\mathbf{r})$, of the core material is

$$
k-k_{0}=\int \delta \rho w_{c}(r) d V+\ldots
$$

Change in the reactivity caused by changes at the interface of the core and the blanket still has to be evaluated. Thus ellipsis (...) here stands for these often very substantial terms. If the change of density is caused by motion of the material,

$$
\delta \rho=\nabla \cdot(\rho \mathrm{u})
$$

where $u$ is the displacement. Substituting this relation into the preceding one and integrating by parts over the whole core, we see that

$$
k-k_{0}=\int \rho u \cdot \nabla w_{c} d V+\cdots
$$

Now, taking the second derivative with time and substituting for $\rho \ddot{u}$ its value from the equation of motion:

$$
\rho \ddot{u}=-\nabla p,
$$

where $\mathrm{p}$ is the "gage" pressure, we have

$$
\ddot{k}=-\int \nabla_{\mathrm{p}} \cdot \nabla_{\mathrm{w}_{\mathrm{c}}} \mathrm{dV}+\ldots
$$

Integrating by parts, we obtain

$$
\ddot{\mathrm{k}}=\int \mathrm{p}\left(\nabla^{2} \mathrm{w}_{\mathrm{C}}\right) \mathrm{dV}-\int \mathrm{p} \nabla_{\mathrm{w}_{\mathrm{C}}} \cdot \mathrm{d} S+\ldots,
$$

where dS is an element of surface in the core very close to the blanket. 
Now we will consider the reduction of reactivity at the interface of the core and the blanket. We will assume that the velocity of wave propagation is very small. Then, if the pressure inside the core is $p$, the pressure at the interface has dropped to an intermediate value

$$
p_{i}=p \frac{\rho_{b} c_{b}}{\rho_{b} c_{b}+\rho c} \text {, }
$$

where $\rho c$ and $\rho b c b$ are wave impedances of the core and of the blanket. In the region of intermediate pressure, the core and blanket particles are traveling at the speed

$$
\dot{\mathrm{u}}=\frac{\mathrm{p}}{\rho_{\mathrm{b}} \mathrm{cb}+\rho_{\mathrm{c}}} \text {, }
$$

and the region itself is increasing with appropriate velocity into the core and into the blanket. Thus the motion of core part increases the first derivative of reactivity at the rate

$$
\int \rho \dot{u} \cdot \nabla w_{c} c d S=\int p \frac{\rho c}{\rho_{b} c_{b}+\rho c} \nabla_{w_{c}} \cdot d S
$$

and motion of the blanket

$$
\int \rho_{\mathrm{b}} \dot{\mathrm{u}} \cdot \nabla_{\mathrm{w}_{\mathrm{b}} \mathrm{c}_{\mathrm{b}} \mathrm{dS}}=\int \mathrm{p} \frac{\rho_{\mathrm{b}} \mathrm{c}_{\mathrm{b}}}{\rho_{\mathrm{b}} \mathrm{c}_{\mathrm{b}}+\rho_{\mathrm{c}}} \nabla_{\mathrm{wb}} \cdot \mathrm{dS} .
$$

The sum of these two contributions is

$$
\int p \nabla w_{c} \cdot d S-\int p \frac{\rho_{b} c_{b}}{\rho_{b} c_{b}+\rho c}\left(\nabla w_{c}-\nabla_{w_{b}}\right) \cdot d S
$$

Upon adding this to Eq. (3), we obtain the total reactivity reduction as

$$
\ddot{k}=\int p\left(\nabla^{2} w_{c}\right) d V-\int p \frac{\rho_{b} c_{b}}{\rho_{b} c_{b}+\rho c}\left(\nabla w_{c}-\nabla w_{b}\right) \cdot d S \text {. }
$$

If the gradients of worth are the same at the interface for core and blanket materials, the second term vanishes and we have quite a simple expression for the reactivity reduction.

Previously, (2) we have derived an equivalent expression in terms of the flux in the diffusion-theory formulation. Analytically, the approach was even more simple and general, since we did not have to define separately worth coefficients for the core and blanket, and did not need to treat 
the interfaces differently from the rest of the reactor. In the one-group treatment, we have expressed reactivity change by Eq. (18) of Ref. (2):

$$
\begin{aligned}
\mathrm{k}-\mathrm{k}_{0}= & \left.\int \rho \mathrm{u} \cdot\left\{\frac{2}{\rho}(\nabla D)(\nabla \Phi)^{2}+\frac{\mathrm{D}}{\rho} \nabla(\nabla \Phi)^{2}-\frac{2}{\rho}(\nabla \Phi) \nabla \cdot(\mathrm{D} \nabla \Phi)\right\} \mathrm{dV}\right) \\
& \int \nu \Sigma_{\mathrm{f}} \Phi^{2} \mathrm{dV} .
\end{aligned}
$$

This equation, which can be written in a form similar to Eq. (1),

$$
\mathrm{k}-\mathrm{k}_{0}=\int \rho \mathrm{u} \cdot \mathrm{fdV},
$$

was valid over the whole reactor, even if it is composed of different materials. However, instead of $\nabla_{w_{C}}$ of Eq. (1), we had

$$
\mathbf{f}=\frac{1}{(D \rho)} \cdot\left\{\nabla(D \nabla \Phi)^{2}-2(D \nabla \Phi) \nabla \cdot(D \nabla \Phi)\right\} / \int \nu \Sigma_{f} \Phi^{2} d V
$$

Now, applying to Eq. (6) the same procedure as we have used in deriving Eq. (3), but taking the volume of the whole reactor for our domain of integration, we obtain

$$
\ddot{k}=\int p(\nabla \cdot f) d V
$$

Since pressure is being generated only in the core, we subdivide our domain of integration into three regions: interior of the core, a pillbox-shaped volume enclosing the interface, and the rest of the blanket. The integral over the blanket vanishes if the velocity of wave propagation is small enough. The integral over the pillbox does not if $f$ is discontinuous (and if the pressure wave has reached the edge of the core). Upon evaluating the latter by means of the divergence theorem, we obtain

$$
\ddot{k}=\int_{\operatorname{cor} e} p(\nabla \cdot f) d V-\int p_{i}\left(f_{C}-f_{b}\right) \cdot d S,
$$

where $p_{i}$ is the pressure at the interface, and $f_{c}$ and $f_{b}$ are the values of function $f$ on the core and the blanket side at this location. It is obvious that the surface integral may contribute a considerable fraction of the reactivity reduction if $\left(f_{C}-f_{b}\right)$ is not small. Equation (9) is equivalent to Eq. (5) if pressure at the interface is assumed to be given by Eq. (4) and the reactor core is composed of one distinct material, the reactor blanket of another. However, Eqs. (8) and (9) are valid also when compositions as well as the density of core and blanket vary spatially. If the core is 
composed of several layers of different materials, we still can as sume that $f=\nabla w$ within each laye $x$ for evaluation of Eq. (8). But we have to add an appropriate surface term, as in Eq. (9). An approximation procedure, as later in Eq. (14), would be completely wrong for such case.

It is interesting to note that the expression for f, Eq. (7) simplifies considerably for a one-dimensional reactor. For a spherical reactor it becomes equal to

$$
f=-\frac{4}{r} \frac{1}{(D \rho)}\left(D \frac{d \Phi}{d r}\right)^{2} / \int \nu \Sigma_{f} \tau^{2} d V
$$

for an infinite cylindrical reactor

$$
f=-\frac{2}{r} \frac{1}{(D \rho)}\left(D \frac{d \Phi}{d r}\right)^{2} / \int \nu \Sigma_{f} q^{2} d V
$$

and it vanishes for a plane reactor.

In the derivation of Eqs. (5) and (9), no mechanism of pressure generation was mentioned and no assumption about its propagation has been made, except in the surface term. Bethe and Tait(1) have assumed that the pressure in this integral can be calculated adequately by neglecting local expansion and propagation of the pressure. This assumption, of course, usually will not give correct local pressures, but it is quite satisfactory for evaluation of the volume integral in Eq. (8) if $(\nabla \cdot f)$ can be considered roughly constant and if nonlinear effects in the propagation of the pressure are not too prominent. (6) In this case the integral $\int$ pdV does not change much during the time of power generation due to such effects. In actual situations, however, $(\nabla \cdot f)$ usually decreases upon going from the center outwards and $\int \mathrm{pdV}$ decreases as time flows by. Thus, a smaller reactivity reduction results and a higher energy yield is obtained. Previously, (2) we have estimated $\nabla \cdot f$ by approximating the flux with a parabola:

$$
\Phi=1-\left(q r^{2} / b^{2}\right) \quad, \quad 0<r<b \quad .
$$

Using this flux and assuming that the microscopic cross sections are constant in the core, we see from Eq. (10) that for a spherical reactor

$$
(\nabla \cdot f)=\frac{48 q^{2} F}{4 \pi \Sigma \operatorname{tr} \nu \Sigma_{f} b^{7}\left[1-(6 q / 5)+\left(3 q^{2} / 7\right)\right] \rho_{c}} \text {, }
$$

where

$$
F=\int_{0}^{b} \nu \Sigma_{f^{2}} \Phi^{2} r^{2} d r / \int_{0}^{\infty} \nu \Sigma_{£} \Phi^{2} r^{2} d r
$$


$b$ is the radius of the core, $\Sigma_{t r}$ and $\Sigma_{f}$ in Eq. (12) are the transport and fission cross sections in the core, and $\rho_{C}$ is the density of core material. In the previous (2) derivation of this constant, we have committed an error in integration, as has been pointed out to us by A. P. Schmitt. ( $)$ Thus, to be consistent with the initial one-group parabolic flux assumption, we should have $\left[1-(6 q / 5)+\left(3 q^{2} / 7\right)\right]$ instead of $(1-0.6 q)$ in Eqs. (21), (23), (28), (30), (32a) and (33a) of that paper. An average value of $\nabla^{2} w_{c}$ can be estimated also by means of other experimental or multigroup-calculated results. If, for a spherical reactor, we assume that $\nabla^{2} w_{C}$ is constant, we see upon integrating that

$$
\nabla w_{C}=(1 / 3)\left(\nabla^{2} w_{C}\right) r
$$

and, on integrating again,

$$
w(b)-w(0)=(1 / 6)\left(\nabla^{2} w_{c}\right) b^{2}
$$

Thus $\nabla^{2} w_{C}$ can be estimated from the difference of core material worth at the center and at the interface. Similarly, another average value of $\nabla^{2} w_{C}$ can be obtained if we know the reactivity change for a uniform expansion of the core alone $(-b \Delta k / \Delta b)$. Then, substituting Eq. (13) into Eq. (1), we obtain

$$
\nabla^{2} w_{c}=-\left(-\frac{b \Delta k}{\Delta b}\right) \frac{15}{4 \pi} \frac{1}{b^{5} \rho_{c}}
$$

\section{B. Solution of Kinetic Equations}

In the preceding section, reactivity reduction ( $\mathrm{k}$ more precisely) has been expressed in terms of pressures generated. When excess reactivity is small and period is short, the integral in Eq. (8) can be evaluated assuming that the power maintains the same spatial shape $N(r)$, and that the energy density e generated at each place,

$$
e(r, t)=Q(t) N(r),
$$

is used to raise pressure without significant expansion:

$$
p=p\left(e, \rho=\rho_{c}\right)
$$

Substituting the pressure obtained in this way into Eq. (8) and performing integrations, we obtain $k$ as a function of energy input $Q(t)$. The actual form of this function depends on particular assumptions about $\nabla \cdot f$, power shape $N$, and connection between pressure and energy density at the initial density of the core material. 
In this section we report calculations performed using the same assumptions as in the original Bethe-Tait paper(1): $\nabla \cdot f$ is constant in the core and small blanket region surrounding it; power density is parabolic in the core and vanishes in the blanket:

$$
\begin{array}{ll}
N=1-\left(q r^{2} / b^{2}\right) & \text { for } r<b \\
N=0 & \text { for } r>b \quad
\end{array}
$$

pressure is negligible below a certain threshold energy density $Q^{*}$ and increases linearly thereafter:

$$
\begin{array}{ll}
p\left(e, \rho_{C}\right)=0 & \text { for } e<Q^{*} \\
p\left(e, \rho_{C}\right)=(\gamma-1) \rho_{C}\left(e-Q^{*}\right), & \text { for } e>Q^{*} .
\end{array}
$$

We have also performed calculations for a simpler set of assumptions: $\nabla \cdot f$ is constant, power shape is arbitrary, and pressure is proportional to the $n^{\text {th }}$ power of energy-density input. The last assumption makes $\ddot{k}$ proportional to the $n^{\text {th }}$ power of the energy input. Numerical calculations for an excursion starting with step reactivity input at negligible power yield values of pressure averaged over the core. Values, calculated in a later section of this paper, of highest average pressure are given in Table III. An approximate value is given by Eq. (22). The energy generated in such case can easily be determined from the highest average pressure if the proportionality constant in the equation of state and power shape $N$ are known.

If $\nabla \cdot f$ is constant in the core and at the interface $f_{b}=f_{C}$, then $\ddot{k}$ is proportional to the pressure integrated over the volume of the core. When the same equation of state is valid throughout the core, it is convenient to perform integrations over the surfaces of constant $\mathrm{N}$ before using the equation of state. For a spherical reactor and a parabolic shape of the power density given by Eq. (15), this is equivalent to the use of $N$ as the variable of integration instead of $r$. Thus, $\vec{k}$ can be written as

$$
\ddot{k}=(\nabla \cdot f) 2 \pi b^{3} q^{-3 / 2} \int_{1-q}^{1} p(N, Q) \sqrt{1-N d N}
$$

Now, if we use the equation of state (16) and perform integration over $N$ we obtain 3 analytical expressions for $\ddot{k}$, depending upon whether the thres hold energy density has been reached at all, in part, or in the whole core: 


$$
\left.\begin{array}{l}
\ddot{k}=0, \text { for } Q<Q^{*} \\
\ddot{k}=-\left(\frac{1}{x} \frac{k_{0}^{3}}{l^{2}}\right) \frac{Q}{Q^{*}}\left(1-\frac{Q^{*}}{Q}\right)^{5 / 2}, \text { for } Q^{*}<Q<Q^{*}(1-q)^{-1}, \\
\ddot{k}=-\left(\frac{1}{x} \frac{k_{0}^{3}}{l^{2}}\right) \frac{5}{2} q^{3 / 2}\left[(1-0.6 q) \frac{Q}{Q^{*}}-1\right], \text { for } Q>Q^{*}(1-q)^{-1}
\end{array}\right\} \text { (17) }
$$

where $1 / x$ is an abbreviation for

$$
\frac{1}{x}=(-\nabla \cdot f)\left(\frac{4 \pi b^{3}}{3}\right)\left(\frac{2}{5} q^{-3 / 2}\right)(\gamma-1) \rho_{c} Q^{*} \frac{l^{2}}{k_{0}^{3}} .
$$

This is a fairly simple expression for $\ddot{k}$ as a function of the energy generated. It is quite clear, however, that, starting with Eq. (9), we can evaluate $\ddot{k}$ for nonconstant $\nabla$. $f$ and for any given power shape and equation of state in the core. Provided we calculate pressures neglecting local expansion and wave propagation, we would end up with an expression for $\ddot{k}$ as a function of energy input, although the expression may be more complex than Eq. (17). Thus one has to consider that the assumptions of constant $\nabla \cdot f$, spherical geometry, parabolic power shape, and simple equation of state for core material are only nonessential simplifications of the BetheTait approach.

If only prompt neutrons are to be considered, the connection between power and reactivity is given by

$$
k=\ell \frac{d}{d t} \ln \dot{Q}
$$

Equations (19) and (17) form 2 coupled, second-order differential equations. These equations were transformed into 4 first-order differential equations in which $k / k_{0}, l k / k_{0}^{2}, Q / Q^{*}$, and $\ln \left(\ell Q^{0} / Q^{*} k_{0}\right)$ are dependent variables. A logarithm of the quantity, proportional to the power has been chosen in preference to the quantity itself in order to assure convergence of energy yield at large values of time. We have measured time in terms of $\ell / k_{0}$ and reactivity in terms of step reactivity input $k_{0}$. Thus the initial conditions of the dependent variables, for an excursion starting with negligible power, do not depend on any parameters. At the beginning of the numerical calculation, our dependent variables were equal to $1,0,1$, and 0 , respectively.

Equation (19) also does not involve any parameters. Equation (17) depends only on $x$ and $q$. Numerical solution of these equations has been performed on the LGP-30 by means of the Runge-Kutta routine. Calculations have been performed with several choices of elementary time interval. 
It had to be made quite small for small $\mathrm{x}$ in order to prevent an unsatisfactory numerical error. Final energies for several values of $\mathrm{x}$ and $\mathrm{q}$ are displayed in Table I and Fig. 1. Calculations for larger and smaller values of $\mathrm{x}$ have also been performed. Determining asymptotes graphically, we have seen that for large values of $x$ the yield approaches slowly to

$$
\frac{Q-Q^{*}}{Q^{*}} \rightarrow \frac{17}{15} \frac{2}{5} \frac{q^{-3 / 2}}{1-0.6 q} \times
$$

whereas for small values of $\mathrm{x}$

$$
\frac{Q-Q^{*}}{Q^{*}} \rightarrow \frac{9}{4} x^{5 / 27}
$$

Table I

RATIO OF EXCESS ENERGY TO THRESHOLD ENERGY,
$\left(\mathrm{Q}-\mathrm{Q}^{*}\right) / \mathrm{Q}^{*}$, IN SIMPLE BETHE-TAIT CALCULATION

\begin{tabular}{|l|l|l|l|l|}
\hline$x$ & 1 & 0.7 & 0.5 & 0.3 \\
\hline $10^{-5}$ & 0.2981 & 0.2981 & 0.2981 & 0.2981 \\
$10^{-4}$ & 0.4902 & 0.4902 & 0.4902 & 0.4902 \\
$10^{-3}$ & 0.8431 & 0.8431 & 0.8431 & 0.8487 \\
$10^{-2}$ & 1.561 & 1.561 & 1.562 & 1.633 \\
$10^{-1}$ & 3.267 & 3.268 & 3.325 & 3.774 \\
$10^{0}$ & 8.398 & 8.461 & 9.090 & 11.69 \\
$10^{1}$ & 29.82 & 31.07 & 36.47 & 54.60 \\
$10^{2}$ & 160.5 & 176.9 & 226.7 & 384.6 \\
\hline
\end{tabular}

In Fig. 2 we have also plotted as solid curves the "widths" of power bursts obtained in Bethe-Tait calculations. The "width" has been defined as the ratio of the integrated power (energy yield $E_{T}$ ) to the maximum power $P_{\max }$ reached and measured in terms of the initial period $\left(l / k_{0}\right)$ : $\mathrm{k}_{0} \mathrm{E}_{\mathrm{T}} / \ell \mathrm{P}_{\max }$. In Bethe-Tait calculations, the "width" increases from one at very small values of $\mathrm{x}$ up to 2.90 for very large values of $\mathrm{x}$. Open dots in Fig. 2 represent the widths of power bursts obtained in AX-I calculations described in Section III-E and Table VI.

C. Excess Energy and an Upper Bound for Mechanical Work

In the derivation of formula (17) the energy density at the center, $Q$, has been used as a parameter, and the ratio of this energy density over 
threshold to threshold energy density is displayed in Table I and Fig. 1. However, since power maintains spatially a nearly constant shape, this ratio is roughly the same as the ratio of total energy in excess of threshold, $E_{\text {ex }}$, to the threshold energy. The latter, $E^{*}$, is understood as the amount of energy generated from the beginning of the excursion, at negligible power, till the incipience of the mechanism for reactivity reduction, when the energy density reaches threshold $Q^{*}$ at the center:

$$
E^{*}=(4 \pi / 3) b^{3} \rho_{C} Q^{*} \cdot(1-0.6 q),
$$

if energy generated in the blanket is negligible. Only integrated quantities $E_{\text {ex }}$ and $E^{*}$, of course, preserve their significance when we allow expansion during heat generation, as in Chapter III. Energy in excess of threshold Eex by itself, however, does not determine energy available for mechanical work. The need to emphasize this has been called to the author's attention by H. A. Bethe. (11)

An upper bound for energy available for mechanical work can be estimated easily by assuming that the energy-density distribution by the end of heat generation still has a parabolic shape and that the threshold energy density does not increase with specific volume. Then the energy density above threshold energy density,

$$
Q\left[1-\left(q r^{2} / b^{2}\right)\right]-Q^{*},
$$

could be converted to work in an isentropic expansion. Simple spatial integration of the above expression gives an upper bound for mechanical work:

$$
\left.\begin{array}{rl}
W_{\max }= & \frac{4 \pi}{3} b^{3} \rho_{c} Q^{*}\left(\frac{Q-Q^{*}}{Q^{*}}\right)^{5 / 2}\left(\frac{Q^{*}}{q Q}\right)^{3 / 2} \frac{2}{5} \\
& \text { if } Q^{*}<Q<Q^{*}(1-q)^{-1} ; \\
W_{\max }= & \frac{4 \pi}{3} b^{3} \rho_{c} Q^{*}\left[\frac{Q}{Q^{*}}(1-0.6 q)-1\right] \\
& \text { if } Q>Q^{*}(1-q)^{-1}
\end{array}\right\}
$$

A dimensionless quantity, $3 q^{3 / 2} W_{\max } / 4 \pi b^{3} \rho_{c} Q^{*}$, has been evaluated by means of Eq. (21) and Table I, and the results are presented in Table II and Fig. 3. For small $\mathrm{x}$ the curves for various q's coincide; for large $\mathrm{x}$, the curves have a common asymptote. Using the previous expression for $\left(Q-Q^{*}\right) / Q^{*}$, we see that

$$
\frac{3 q^{3 / 2} W_{\max }}{4 \pi b^{3} \rho_{c} Q^{*}} \rightarrow 0.45 x \text { for } x>1
$$


Table II

AN UPPER BOUND FOR MAXIMUM WORK, $3 \mathrm{q}^{3 / 2} \mathrm{~W}_{\mathrm{max}} / 4 \pi \mathrm{b}^{3} \rho_{\mathrm{c}} \mathrm{Q}^{*}$, AS GIVEN BY EQ. (2I)

\begin{tabular}{|l|l|l|l|l|}
\hline $\mathrm{q}$ & 1 & 0.7 & 0.5 & 0.3 \\
\hline $10^{-5}$ & 0.0131 & 0.0131 & 0.0131 & 0.0131 \\
$10^{-4}$ & 0.0370 & 0.0370 & 0.0370 & 0.0365 \\
$10^{-3}$ & 0.104 & 0.104 & 0.104 & 0.0848 \\
$10^{-2}$ & 0.297 & 0.297 & 0.281 & 0.190 \\
$10^{-1}$ & 0.875 & 0.864 & 0.717 & 0.479 \\
$10^{0}$ & 2.84 & 2.63 & 2.14 & 1.55 \\
$10^{1}$ & 11.4 & 10.3 & 8.92 & 7.33 \\
$10^{2}$ & 63.6 & 59.8 & 56.0 & 51.8 \\
\hline
\end{tabular}

This simple estimate of upper bound for energy available for mechanical work, however, usually is too large, particularly for yields of low energy, since threshold energy density increases with increasing specific volume. A better estimate can be obtained if the equation of state for isentropic expansion is known. For example, if is entropic expansion is described by Eq. (16a) of Appendix $A$ and $Q \backslash Q^{*}(1-q)^{-1}$, one finds that $W_{\text {max }}$ obtained from Fig. 3 or Table II should be multiplied by a function $F$ of $\beta^{\prime}\left(Q-Q^{*}\right) /\left(-T^{\prime} A^{\prime}\right)$. This function is nearly equal to one if values of the argument are larger than 10 , but

$$
F=\frac{2}{7} \frac{\beta^{\prime}\left(Q-Q^{*}\right)}{-\tau^{\prime} A^{\prime}}\left[1-\frac{4}{9} \frac{\beta^{\prime}\left(Q-Q^{*}\right)}{-\tau^{\prime} \Lambda^{\prime}}+\ldots\right] \text {, if } \frac{\beta^{\prime}\left(Q-Q^{*}\right)}{-\tau^{\prime} A^{\prime}} 1 \text {. }
$$

D. Approximate Solutions of Kinetic Equations

Previously, $(\underline{1,6})$ some approximate solutions of coupled equations, Eqs. (17) and (19), have been made on the assumption that the heat input $Q$ continues rising exponentially; the reactivity is then calculated from Eq. (17) till it vanishes. The value of $Q$ at this time was considered a fair approximation to the total energy generated during a nuclear incident, including that generated during the time in which $k_{\text {ex }}$ is negative and the power rate of generation is falling to zero. It is nearly correct for very large values of the argument $x$, since the actual value is only 1.13 of the approximate value. However, for smaller values of the argument this approximation procedure is a serious underestimate. This can be seen by examining Fig. 4, in which the ratio of the actual value of excess 
energy (obtained by numerical program of Section II B) to the approximate value $\mathrm{BT} / \mathrm{BT}^{\prime}$ has been plotted for $\mathrm{q}=1$ and $\mathrm{q}=0.483$. Calculations based on this cut-off model have been performed with the IBM-704. In these calculations AX-I has been modified, so that the heat generation rate continues to rise exponentially. The program, called AX-I', terminated the calculation when the excess reactivity $k_{0}$ was reduced to zero. The ratio of the results for excess energy, $\mathrm{AX}-\mathrm{I} / \mathrm{AX}-\mathrm{I}^{\prime}$, has been plotted in Fig. 5 . One observes that in both figures a curious inflection region in the curve is obtained after the zone of pressure generation reaches the interface between the core and the blanket.

A better approximate solution of the coupled equations, Eqs. (17) and (19), could be obtained by using the "long delay" approximation for reactivity reduction. Then one determines reactivity, from Eq. (17), assuming that the heat input continues to rise exponentially with time. Using this reactivity one finds numerically power and energy yield from Eq. (19). This approximation has been used by R. D. Nicholson. (4)

We have used this method to calculate the yield of an excursion when the pressure is proportional to $n^{\text {th }}$ power of heat input. Assuming that the heat input continues to rise exponentially, we see that reactivity reduction calculated from Eq. (8) also increases exponentially, at a different rate. Using reactivity found this way, we can integrate Eq. (19) for power and total energy yield exactly.(10) And we obtain, that the final value for "dimensionless" pressure averaged over the core is

$$
\left[\bar{p}_{\max }\right]=(-\nabla \cdot f) \bar{p}_{\max }(4 \pi / 3) b^{3}\left(\ell^{2} / k_{0}^{3}\right) \approx n^{3}\left[\Gamma\left(1+\frac{1}{n}\right)\right]^{n} ;
$$

$\bar{p}_{\mathrm{max}}$ is the highest average pressure in the core.

Exact solutions of kinetic equations were also obtained by numerical integration. They are compared with these approximate values in Table III. One notices that, for all $\mathrm{n}$, the long-delay approximation underestimates pressure yield by about 13\%. Thus it is likely that long-delay approximation will underestimate average peak pressure only by a little also for other connection between pressure and heat input. On the other hand, the original Bethe-Tait approximation would give $n^{2}$ for the right side of Eq. (22) and commit an appreciable error if $\mathrm{n}$ is much different from 1 .

Table III

THE PEAK OF THE (DIMENSIONLESS) AVERAGE CORE PRESSURE, EOS. (22) OR (8b), IF PRESSURE IS PROPORTIONAL TO $\mathrm{n}^{\text {th }}$ POWER OF HEAT INPUT

\begin{tabular}{|c|c|c|c|c|c|}
\hline$n$ & {$[\bar{p}]$} & $n^{3}\left[\Gamma\left(1+\frac{1}{n}\right)\right]^{n}$ & $n$ & {$[\bar{p}]$} & $n^{3}\left[\Gamma\left(1+\frac{1}{n}\right)\right]^{n}$ \\
\hline 0.5 & 0.202 & 0.177 & 3.0 & 21.9 & 19.2 \\
1.0 & 1.13 & 1.00 & 3.5 & 33.4 & 29.5 \\
1.5 & 3.26 & 2.89 & 4.0 & 48.6 & 42.9 \\
2.0 & 7.08 & 6.30 & 4.5 & 68.7 & 60.7 \\
2.5 & 13.1 & 11.54 & 5.0 & 91.7 & 81.6 \\
\hline
\end{tabular}




\section{AX-I CALCULATION}

In the preceding chapter, investigation of an excursion by the generalized Bethe-Tait method was described. In Section II-A we have derived a general formula for the reactivity reduction based on perturbation theory and Newton's equation of motion. (2) This general formula gave an expression for reactivity reduction in terms of pressures generated and was evaluated in terms of energy input neglecting expansion of volume elencents. Detailed evaluation has been reported only for a special assumption of $\nabla \cdot f$, shape of power density $N$, and equation of state $\mathrm{p}\left(\mathrm{e}, \rho_{\mathrm{C}}\right)$. It has yielded $\mathrm{Eq}$. (17). The resulting kinetic equations have been solved on the LGP-30 by means of a simple numerical program. For different assumptions regarding $\nabla \cdot f, N$, and $p\left(e, \rho_{c}\right)$, Eq. (17) would have a different form, but investigation of an excursion could be as easily performed.

It is obvious that, when $k_{0}$ is very small, perturbation theory is valid. When $l / k_{0}$ is so small that the pressure wave does not have time to propagate considerable distance, evaluation of pressure neglecting expansion is correct. Thus the Bethe-Tait theory should be considered correct for a limiting case in which the nuclear parameter $k_{0}$ and $\ell$ become vanishingly small, $k_{0}^{3} / \ell^{2}$ staying finite. In actual situations, however, wave propagation covers a considerable distance during an. excursion. It can be shown (6) that for $\nabla \cdot f$ constant and propagation of pressure linear the effect of wave propagation on reactivity reduction is negligible. Wave propagation usually involves nonlinear effects and $\nabla \cdot f$ cannot be considered constant everywhere. More complicated calculations are needed to assess deviations from Bethe-Tait theory.

This chapter reports several sets of such calculations. They were performed by means of a numerical program AX-I. (3) This program follows exactly the progress of a prompt excursion in a spherical reactor. In it, neutron flux, power, pressure, and displacements are calculated repeatedly at adequately short intervals of time. In our calculations, the step input of reactivity has been changed while the lifetime velocity of wave propagation and other constants have also been varied. Results have been arranged so that a comparison with Bethe-Tait theory can be made conveniently. In all the cases considered, conditions that lead towards larger "distance of wave propagation" during excursion result in a larger deviation from the Bethe-Tait calculation. Energy yields are always somewhat larger and peak pressures obtained are considerably lower. Some results of these calculations have been presented earlier in a preliminary fashion. (12)

In contrast with the semi-analytical Bethe-Tait method, the results of $\mathrm{AX}-\mathrm{I}$ calculations have been obtained in dimensional form, keeping some input parameters the same. The results, however, can be used. 
to describe similar excursions. In Appendix B some scaling laws, based on proportionality of excess reactivity to the displacements, have been substantiated by numerical calculations. According to them, the progress of the burst is identical if the equations of state are the same in a system of units in which time is measured in terms of initial period and change of density in units proportional to the step input of reactivity.

A. General Description of the Code

Given a spherically symmetric, superprompt critical system, the program computes the variation in time and space of the specific energy, temperature, pressure, density, and velocity. As a function of time it computes the reactivity (in the form of alpha, the inverse period), the power, the total energy, and the position of the boundaries of the various shells into which the system has been subdivided. All delayed-neutron effects are ignored, and no allowance is made for transfer of heat by conduction or radiation. The input information includes the initial reactivity or geometry, the initial velocities and temperatures of the mass points, the composition and disposition of materials, the appropriate constants of the equation of state, and the microscopic neutron cross sections. For calculational purposes the spherical assembly is divided into a number of hypothetical spherical shells or mass points. The neutronics of this system is calculated in conventional fashion, using the $\mathrm{S}_{4}$ method, thereby providing a power distribution across the radial network, as well as the alpha of the system. From the neutronics calculation one goes to the thermodynamics and hydrodynamics portion of the code to calculate the variation of power, temperature, pressure, density, and velocity with time.

The calculation proceeds initially like the usual $S_{n}$ calculation. After computing average cross sections for each of the spherical shells in the mixture code, the program proceeds either to a calculation of alpha $\left(k_{e x} / l\right)$ for the specified configuration, or to a scaling of the reactor radii to provide the alpha originally specified. Before proceeding to the hydrodynamics, the code also computes $k_{\text {eff }}\left(=1+k_{0}\right)$ for the initially converged configuration, if so requested.

Then, for one or more short time intervals $\Delta t$, alpha is considered to remain constant while the power varies as exp $\alpha \Delta t$. From the pressure gradients in the system, the average accelerations of the mass points are computed and, hence, the new velocities at the end of the time interval. These, in turn, lead to the new radial positions of each shell boundary at the end of the time interval. The solution is performed in a Lagrangian coordinate system, i.e., the mesh is embedded in the material and follows it along throughout its motion. 
During the time interval, energy is added to the system (the average power times $\Delta t$ ) and this is distributed among the shells in accord with the previously calculated fission distribution. If allowance is made for the work done by or on a shell in expansion or compression, the net change in internal energy is computed, and from the internal energy a new pressure and temperature are obtained. The so-called viscous pressure, obtained by a mathematical procedure of von Neumann and Richtmyer, $(7,3)$ is included to permit thermodynamic and hydrodynamic calculations in the presence of steep shock front. Hence, the total pressure used is the sum of the hydrodynamic pressure, given by the equation of state, and the synthetic viscous pressure $\mathrm{p}_{\mathrm{V}}$ :

$$
p_{v}=C_{v p} \frac{1}{\rho}\left(\Delta R \frac{\partial \rho}{\partial t}\right)^{2},
$$

where $\rho$ is the density, $\triangle R$ is the mesh width, and numerical constant $\mathrm{C}_{\mathrm{vp}}$ is usually taken to be between 1.5 and 2 .

When calculation of thermo- and hydrodynamic changes during the time interval $\Delta \mathrm{t}$ is complete (a hydrocycle), a series of tests is $\mathrm{r}$ un and the program proceeds with another hydrocycle or goes back to the neutronics calculation. To control the pace of a problem, the code continually examines the magnitude or rate of change of certain crucial parameters, and varies $\Delta t$ of a hydrocycle or the number of hydrocycles per neutron cycle accordingly. This latter number begins at unity and is allowed to build up gradually if the forces present are not changing alpha too rapidly or modifying the density of a mass point radically. When the power variation in a hydrocycle, or the change in alpha between neutron cycles, gets so large as to damage the accuracy of the solution, the pace of the calculation is slowed automatically - or stopped in extreme cases.

B. Common Procedure Used in Present Study

In the formulation of the AX-I code (see Appendix A) the hydrostatic pressure $p$ depends linearly on the temperature $\theta$ and the density $\rho:$

$$
p=\tau+\beta \theta+\alpha \rho
$$

if the temperature and density are sufficiently large. In all the calculations, however, $\tau$ was chosen to have a rather large negative value. The hydrostatic pressure was set to zero by the code whenever above expression yielded a negative value. All the calculations have been started at low temperature and density. Thus one needed to produce an appreciable amount of energy density to generate nonvanishing pressure in the core. The specific heat at constant density has been assumed to vary linearly with temperature: 


$$
C_{V}=\left(\frac{\partial e}{\partial \theta}\right)_{V}=A+B \theta
$$

A system of units: $\mathrm{g}, \mathrm{cm}, \mu \mathrm{sec}, \mathrm{kev}$, has been used. Thus, density has been measured in $\mathrm{g} / \mathrm{cm}^{3}$, temperature in kev, pressure in megabar $\left(10^{12}\right.$ dyne/ $\left.\mathrm{cm}^{2}\right)$, energy density e in $10^{12} \mathrm{erg} / \mathrm{g}$.

Since, during the time heat is being generated in an excursion, there is no appreciable thermal conduction, information about temperature is superfluous and only the relation connecting pressure with internal energy e and density is needed. Eliminating $\theta$ we obtain Eq. (6a) of Appendix A. This relation,

$$
e=\frac{A}{\beta}(p-\tau-\alpha \rho)+\frac{B}{2 \beta^{2}}(p-\tau-\alpha \rho)^{2}-\frac{\tau}{\rho}+\alpha \ln p+\text { const }
$$

is fairly complex, involving $5 \mathrm{free}$ constants. By choosing them judiciously, we can hope to approximate even a fairly complicated equation of state governing the actual physical situation during an excursion.

In the initial calculations, reported in Section III-C, the constants of the equation of state, with the exception of $\tau$, have been chosen to be the same as used in early calculations by Stratton. $(8,9)$ Using his set of constants, Stratton was able to represent successfully the burst of Godiva.

The AX-I code does not allow for shearing stress. Thus, for solids Stratton had to make the stress tensor diagonal artificially. A modified code called Hatchet (13) (suitable for a reactor composed of spherically symmetric shells) that allows tangential strain is now available. However, based on Stratton's success, no claim can be made that the same numerical values are applicable to a reactor with voids, differing much in density from Godiva. Indeed, more sophisticated considerations of uranium properties point to the contrary. (9) So in the present set of calculations some of the parameters have been varied to obtain an indication of how they influence the energy yield in an excursion.

After the initial calculations, some simplifications of the equations of state were made. A few constants pertaining to the behavior of the core material have been kept the same throughout the study (except as noted otherwise). In all the problems reported, the core density has been the same:

$$
\rho_{C}=7.92 \mathrm{~g} / \mathrm{cm}^{3}
$$


Also, the initial rate of pressure rise

$$
\begin{aligned}
\left(\frac{\partial p}{\partial e}\right)_{\rho} & =\frac{\beta}{A+B \theta s p} \\
& =\frac{\beta^{2}}{A \beta-B\left(\tau+\alpha \rho_{c}\right)}=17.815 \frac{\mathrm{g}}{\mathrm{cm}^{3}}
\end{aligned}
$$

has been kept the same throughout the study.

All the calculations have been performed with one-energy group neutrons. The lifetime of the reactor has been varied by varying the velocity of the neutrons. All calculations have been started with the reactor material at rest, with negligible power density, and with initial core temperature at

$$
\theta_{i}=10^{-4} \mathrm{kev}\left(=1,161^{\circ} \mathrm{K}\right) \text {. }
$$

Actually, since in the initial stage the power and energy increase exponentially, some machine time has been saved by assuming an initial energy distribution of proper parabolic shape (and everywhere well below threshold) and starting the calculation with the power equal to the total energy $r i s e$ at this time divided by the initial period $k_{0} / l$. At criticality, the reactor core was about $23.28 \mathrm{~cm}$ in radius and it had about a $20-\mathrm{cm}$ blanket. The computing machine varies linear dimensions of the reactor, keeping composition (density and cross sections) constant to achieve the requested initial inverse period. Hence, in the calculations reported, the radius of the core was somewhat larger, being roughly

$$
\mathrm{b} \approx 23.4 \mathrm{~cm}
$$

The threshold energy (generated before nonvanishing pressure has been reached in the center) was

$$
\mathrm{E}^{*} \approx 2,100 \times 10^{12} \mathrm{ergs}
$$

except as indicated in Section III-D. Since $b$ and $E^{*}$ varied little among the problems, we have not given detailed account of their variation except in Figs. 8, 10, and 11 .

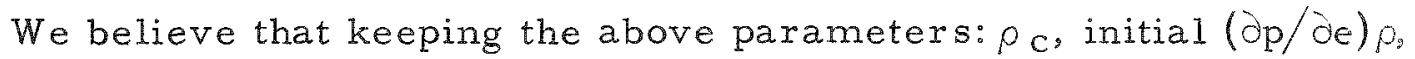
$\mathrm{b}$, and $\mathrm{E}^{*}$, the same and varying lifetime $l$ and reactivity inserted, $k_{0}$. does not restrict much the generality of the study. The belief is based upon arguments advanced in Appendix B. There, relying on plausible assumptions (that during the excursion density variation is small and that 
reactivity is proportional to this variation), we are able to construct solutions that depend only on two of the dimensionless combinations of the above six constants.

C. Variation of Lifetime

In the initial set of calculations, the prompt lifetime was varied by varying the neutron velocity. For an artificially large neutron velocity, $\mathrm{V}_{\mathrm{g}}=1695 \mathrm{~cm} / \mu \mathrm{sec}$, a lifetime of approximately $l \approx 0.024 \mu \mathrm{sec}$ was obtained. Then the neutron velocity was arbitrarily decreased by a factor of 10 and by a factor of 100, resulting in roughly proportional increases in the lifetime.

The core was assumed to be composed of $36 \%$ enriched uranium. Microscopic cross sections (in barns) for $U^{235}$ were taken as

$$
\nu \sigma_{f}=3.75 ; \quad \sigma_{t r}=7.0 ; \quad \sigma_{\text {scatt }}=5.3 .
$$

Cross sections for $\mathrm{U}^{238}$ were chosen to be

$$
\nu \sigma_{f}=0.25 ; \quad \sigma_{t r}=7.0 ; \sigma_{\text {scatt }}=6.7 .
$$

In the blanket no heat generation was allowed, this assumption being indicated by choosing for the blanket material

$$
\nu \sigma_{f}=0 ; \quad \sigma_{t r}=7.0 ; \quad \sigma_{\text {scatt }}=6.8 .
$$

The constants for the equations of state of core material, of density $7.92 \mathrm{~g} / \mathrm{cm}^{3}$, were

$$
\begin{aligned}
& \alpha=0.02873 \mathrm{~cm}^{2} / \mu \mathrm{sec}^{2} ; \quad \beta=278.46 \mathrm{~g} / \mathrm{cm}-\mu \mathrm{sec}^{2}-\mathrm{kev} ; \\
& \tau=-0.3946 \mathrm{~g} / \mathrm{cm}-\mu \mathrm{sec}^{2} ; \quad A=12.163 \mathrm{~cm}^{2} / \mu \mathrm{sec}^{2}-\mathrm{kev} ; \\
& B=5780 \mathrm{~cm}^{2} / \mu \mathrm{sec}^{2}-\mathrm{kev}^{2} .
\end{aligned}
$$

The density of the blanket was chosen to be $15.83 \mathrm{~g} / \mathrm{cm}^{3}$. The constants for the equation of state in the blanket were chosen to be the same as in the core except for $\tau$. The latter has been chosen $\tau=-0.4687189 \mathrm{~g} / \mathrm{cm}-\mu \mathrm{sec}^{2}$ in order to make blanket pressure exactly zero at the initial density and the starting temperature of $5 \times 10^{-5} \mathrm{kev}$. The starting temperature of the core was $\theta_{i}=10^{-4} \mathrm{kev}$, thus requiring

$$
Q^{*}=A\left[-\frac{\tau+\alpha \rho_{C}}{\beta}-\theta_{i}\right]+\frac{1}{2} B\left[\left(-\frac{\tau+\alpha \rho_{C}}{\beta}\right)^{2}-\theta_{i}^{2}\right]=0.007093 \mathrm{~cm}^{2} / \mu \sec ^{2}
$$


for the energy density to start generating pressure. With this set of constants given by Eqs. (29), (30), and (31), the ratio of power at the interface to that at the center was

$$
1-q=0.517 \text {. }
$$

If one computes $\mathrm{x}$ of the Bethe-Tait theory approximately, using Eqs. (18) and (12), while for $(\gamma-1) \rho_{c}$ the initial value of ( $\left.\partial p / \partial e\right) \rho$ is taken, there is obtained

$$
\mathrm{x} \approx 1.10 \times 10^{4} \mu \sec ^{2}\left(k_{0}^{3} / \ell^{2}\right)
$$

The results of these computations are given in Table IV and Figs. 6 and 7 . In these and other figures, dots represent values obtained directly from an IBM-704 computation. Because of the threshold energy density $Q^{*}$, roughly the same amount of energy $E^{*}$ had to be generated in all cases before pressures started to build up and the reactivity reduction became effective. Thus, only the excess over actual threshold energy is given in this and the following tables and figures. The values for pressure, $p_{\max }$, given in this and the following tables are the maximum pressures at the center printed out by IBM-704. For small excursions, the pressure varied considerably between successive print-outs. Thus, the values for pressure given in the tables may not exactly be the maximum pressures reached at the center.

Table IV

VARIATION OF LIFETIME

\begin{tabular}{|c|l|l|r|c|c|}
\hline $\begin{array}{c}\text { Problem } \\
\text { No. }\end{array}$ & $\begin{array}{c}k_{0}^{3} / \ell^{2} \\
\left(\mu \mathrm{sec}^{-2}\right)\end{array}$ & $\begin{array}{c}\ell \\
(\mu \mathrm{sec})\end{array}$ & $\begin{array}{c}\text { Excess Energy } \\
\text { Yield }\left(10^{12} \mathrm{erg}\right)\end{array}$ & $\begin{array}{c}\text { Center Peak } \\
\text { Pressure } \\
(\text { megabar })\end{array}$ & $\begin{array}{c}\text { Maximum } \\
\text { Kinetic } \\
\text { Energy } \\
\left(10^{12} \mathrm{erg}\right)\end{array}$ \\
\hline 1 & $4.609 \times 10^{-10}$ & 0.0206 & 612 & 0.0064 & 1.36 \\
2 & $7.947 \times 10^{-9}$ & 0.0227 & 1,000 & 0.0176 & 9.7 \\
3 & $6.576 \times 10^{-8}$ & 0.0234 & 1,600 & 0.044 & 34.5 \\
4 & $5.387 \times 10^{-7}$ & 0.0240 & 2,770 & 0.106 & 150 \\
5 & $8.602 \times 10^{-6}$ & 0.0246 & 7,076 & 0.295 & 957 \\
6 & $6.950 \times 10^{-5}$ & 0.0248 & 19,450 & 0.709 & 3,540 \\
7 & $2.048 \times 10^{-4}$ & 0.0249 & 38,400 & 1.18 & 8,180 \\
14 & $8.834 \times 10^{-3}$ & 0.0252 & $1,760,000$ & 11.3 & 376,000 \\
15 & $7.143 \times 10^{-2}$ & 0.0255 & $55,228,000$ & 65.7 & $2,134,000$ \\
8 & $8.69 \times 10^{-9}$ & 0.248 & 1,178 & 0.0076 & 4.5 \\
10 & $5.552 \times 10^{-7}$ & 0.248 & 3,050 & 0.0594 & 224 \\
12 & $2.081 \times 10^{-4}$ & 0.253 & 47,000 & 1.056 & 15,160 \\
9 & $8.767 \times 10^{-9}$ & 2.51 & 1,890 & 0.00254 & $1.05 *$ \\
11 & $5.64 \times 10^{-7}$ & 2.52 & 4,470 & 0.0228 & 40 \\
13 & $2.180 \times 10^{-4}$ & 2.66 & 125,000 & 0.755 & 60,440 \\
\hline
\end{tabular}


Comparing with Fig. 1, one notices that, while curves for excess energy yield have roughly the same shape as in the Bethe-Tait case, the pressure decreases faster with decreasing values of $k_{0}^{3} / \ell^{2}$. Also, there exists a considerable lowering of the pressure with increasing lifetime, while the concurrent rise of energy yield is usually of smaller size. In the tables, we have given also the maximum kinetic energies obtained during the run of the problem. However, usually this is not the final value, and further discussion of it is postponed until Section III-G.

D. Variation of Blanket Density

Treating an excursion in the Bethe-Tait manner, one finds that (provided the "microscopic" transport cross sections $\Sigma_{t r} / \rho$ are the same in the core and in the blanket) the energy yield depends only upon the functional relationship between pressure and energy in the core, and is entirely independent of the density and equation of state in the blanket. By rough physical considerations, however, one is inclined to expect a higher damage potential in a reactor with a heavy blanket, since this blanket provides a tamping tending to contain the reactor. The Bethe-Tait approximation asserts that displacements of the blanket reduce reactivity, just like displacements of the core, and the smaller displacement obtained in a heavier blanket is exactly compensated by a correspondingly larger transport cross section of the blanket material. It was of interest to check this by a few numerical calculations.

In this set of problems the same microscopic cross sections were used as in Section III-C. The neutron velocity was $V_{g}=1695 \mathrm{~cm} / \mu \mathrm{sec}$. But 3 quite different blanket densities were chosen, and appropriate enrichments of the core were determined to keep the reactor critical. This procedure led to different power levels at the core and blanket interface relative to the power level at the center, 1 - q, as well as to some change in the lifetime. The equation of state was simplified somewhat by choosing $B=0$, and changing A so that the initial rate of pressure rise remained the same as in Eq. (25). The parameters for the equation of state used were

$$
\begin{array}{rlrl}
\alpha & =0.02873 \mathrm{~cm}^{2} / \mu \mathrm{sec}^{2} ; & \beta & =278.46 \mathrm{~g} / \mathrm{cm}-\mu \mathrm{sec}^{2}-\mathrm{kev} ; \\
\tau_{C} & =-0.3946 \mathrm{~g} / \mathrm{cm}-\mu \mathrm{sec}^{2} ; & A=15.631 \mathrm{~cm}^{2} / \mu \mathrm{sec}^{2}-\mathrm{kev} ; \\
B & =0
\end{array}
$$

The use of these values caused the threshold energy density to be raised to

$$
Q^{*}=0.007815 \mathrm{~cm}^{2} / \mu \mathrm{sec}^{2},
$$

and, because of the variation in $q$, the threshold energy $E^{*}$ has not been constant in this set of the problems. 
The results of these calculations are given in Table V. To compare these results with the predictions of Bethe-Tait theory, we have plotted the results of calculations for $\ell \approx 0.024 \mu \mathrm{sec}$ in the previous section as a solid curve in Fig. 8. The ordinate is ratio of energy in excess of threshold to the threshold energy; the abscissa is $\mathrm{x}$ defined by Eqs. (18) and (12):

$$
x=\left[\frac{5}{32} \frac{\sum_{\operatorname{tr}} \nu \Sigma_{f}\left[1-(6 q / 5)+\left(3 q^{2} / 7\right)\right] b^{2}}{\sqrt{q} F}\right] \frac{b^{2}}{(\gamma-1) Q^{*}} \frac{k_{0}^{3}}{b^{2}} .
$$

The results of the present calculations are indicated in Fig. 8 by the open dots. There is a slight deviation from the smooth curve at low yields. Since the pressure waves at low yields do not reach the blanket before shutdown, this certainly cannot be attributed to a difference in blanket density, but must be attributed to the inaccuracy in assigning $q$ and a slight variation in $\ell$. There is a larger deviation for high-energy yield, but a deviation of this magnitude is also predicted by the Bethe-Tait theory (see Fig. 1) as a consequence of a difference in $q$. Some decrease in energy yield relative to the solid curve for large $x$ can be attributed to the change of equation of state at initial density by making $B$ vanish. Thus we conclude that the density of the blanket has no significant, direct influence for the short lifetimes considered in these calculations. Its influence may be larger for longer lifetimes.

Table V

VARIATION OF BLANKET DENSITY

\begin{tabular}{|c|c|c|c|c|c|c|}
\hline $\begin{array}{c}\text { Problem } \\
\text { No. }\end{array}$ & $\begin{array}{c}k_{0}^{3} / \ell^{2} \\
\left(\mu \mathrm{sec}^{-2}\right)\end{array}$ & $\begin{array}{c}\text { Blanket } \\
\text { Density } \\
\left(\mathrm{g} / \mathrm{cm}^{3}\right)\end{array}$ & $\mathrm{q}$ & $\begin{array}{c}\text { Excess Energy } \\
\text { Yield }\left(10^{12} \text { erg }\right)\end{array}$ & $\begin{array}{c}\text { Center Peak } \\
\text { Pressure } \\
(\text { megabar })\end{array}$ & $\begin{array}{c}\text { Maximum } \\
\text { Kinetic } \\
\text { Energy } \\
\left(10^{12} \text { erg }\right)\end{array}$ \\
\hline 27 & $8.642 \times 10^{-9}$ & 18.5077 & 0.476 & 1,080 & 0.0197 & 11.55 \\
28 & $5.475 \times 10^{-7}$ & 18.5077 & 0.476 & 2,825 & - & - \\
29 & $2.094 \times 10^{-4}$ & 18.5077 & 0.476 & 37,510 & 1.87 & 12,750 \\
30 & $7.003 \times 10^{-9}$ & 7.92 & 0.586 & 870 & 0.0156 & 5.65 \\
31 & $4.45 \times 10^{-7}$ & 7.92 & 0.586 & 2,380 & 0.1008 & 132 \\
32 & $1.665 \times 10^{-4}$ & 7.92 & 0.586 & 23,840 & 1.376 & 8,584 \\
33 & $5.314 \times 10^{-9}$ & 3.96 & 0.691 & 700 & 0.0138 & 3.11 \\
34 & $3.401 \times 10^{-7}$ & 3.96 & 0.691 & 1,840 & 0.0875 & 68.25 \\
35 & $1.257 \times 10^{-4}$ & 3.96 & 0.691 & 16.370 & 1.094 & 5,573 \\
\hline
\end{tabular}

E. Yield as a Function of $k_{0}$ and $k_{0}^{3} / l^{2}$

In the reactor excursions that we are interested in now, excess reactivity is always a small number. Therefore, first-order perturbation treatment can be expected to be valid, and reactivity reduction can be expected to be adequately described by equations of type (8). In a calculation 
of the Bethe-Tait type, Eq. (8) is used with an additional assumption that pressure and acceleration can be adequately calculated by neglecting expansion entirely and using the entire heat input to raise the internal energy. Thus, inertial effects are considered to be of paramount impor tance. Then, for a step input of reactivity, the computation gives energy yield as a function of $k_{0}^{3} / \ell^{2}$.

On the other hand, in some cases, particularly for minor accidents of low-energy yield, it is permissible to neglect inertial effects entirely. Then expansion and corresponding reactivity reduction follow energy input immediately. In such cases, the pressure during an excursion should also be considered negligible. This type of computation for step insertion of reactivity (neglecting delayed neutrons) gives energy yield as a function of $k_{0}$ and entirely independently of the lifetime $l$.

It is obvious that these 2 assumptions represent extreme cases. In actual situations the pressure is not negligible and depends, not only on heat input, but also on concurrent expansion. To demonstrate this, several calculations with different values of $k_{0}^{2} / b^{2}$ and $k_{0}$ were made. In these calculations, enrichment of the core was $44.15 \%$ and the ratio of power at the interface to that in the center was $1-q=0.414$. The equation of state was simplified by choosing $\alpha=B=0$ and the values

$$
\begin{aligned}
& \beta=108.046 \mathrm{~g} / \mathrm{cm}^{\prime} \mu \mathrm{sec}^{2}-\mathrm{kev} ; T=-0.15 \mathrm{~g} / \mathrm{cm}^{2} \mu \mathrm{sec}^{2} ; \\
& A=6.065 \mathrm{~cm}^{2} / \mu \mathrm{sec}^{2}-\mathrm{kev}
\end{aligned}
$$

for the other constants. Thus the threshold energy density becomes $Q^{*}=0.007815 \mathrm{~cm}^{2} / \mu \mathrm{sec}^{2}$ and common constants, Eqs. (25) and (26), of the present study are preserved. The same equation of state was used also for the blanket.

The results are in Table VI. After reduction into dimensionless form, as explained in Appendix $B$, the results for energy yield and center peak pressure are also displayed in Figs. 10 and 11: Here dimensionless variables for arguments have also been used. A quantity proportional to $k_{0}^{3} / l^{2}$, namely, $x$ of Eq. (32), has been plotted as abscissa. A quantity proportional to $k_{0}$, namely, y given by $E q .(6 b)$, was treated as a parameter. One notices that a larger value of $k_{0}$ (for a given $k_{0}^{3} / \ell^{2}$ ) results in a somewhat larger energy yield and a considerably smaller peak pressure. From Fig. 10 one notices that, with decreasing $k_{0}^{3} / l^{2}$, curves of constant $k_{0}$ tend to become horizontal, thus indicating approach to the region where the energy yield becomes a function of $k_{0}$ only, and inertial effects tend to disappear. 
Table VI

VARIATION OF REACTIVITY INPUT

\begin{tabular}{|c|c|c|c|c|c|}
\hline $\begin{array}{c}\text { Problem } \\
\text { No. }\end{array}$ & $\begin{array}{c}\mathrm{k}_{0}^{3} / \ell^{2} \\
\left(\mu \mathrm{sec} \mathrm{c}^{-2}\right)\end{array}$ & $k_{0}$ & $\begin{array}{c}\text { Excess Energy } \\
\text { Yield (1012 erg })\end{array}$ & $\begin{array}{c}\text { Center Peak } \\
\text { Pressure } \\
(\text { megabar })\end{array}$ & $\begin{array}{c}\text { Maximum } \\
\text { Kinetic } \\
\text { Energy } \\
\left(10^{12} \mathrm{erg}\right)\end{array}$ \\
\hline 59 & $7.003 \times 10^{-9}$ & 0.000141 & 853 & 0.0208 & 10.2 \\
170 & $4.45 \times 10^{-7}$ & 0.000141 & 2,338 & 0.1423 & 199. \\
171 & $1.665 \times 10^{-4}$ & 0.000141 & 22,892 & 1.570 & $1,281 . *$ \\
76 & $7.003 \times 10^{-9}$ & 0.000560 & 921 & 0.00895 & 6.17 \\
60 & $4.45 \times 10^{-7}$ & 0.000560 & 2,378 & 0.113 & 58. \\
173 & $1.665 \times 10^{-4}$ & 0.000560 & 23,040 & 1.544 & $4,136 . *$ \\
77 & $7.003 \times 10^{-9}$ & 0.004088 & 1,486 & 0.00479 & 1.68 \\
175 & $4.45 \times 10^{-7}$ & 0.004088 & 2,561 & 0.049 & 179. \\
61 & $1.665 \times 10^{-4}$ & 0.004088 & 23,752 & 1.391 & $8,681 . *$ \\
\hline
\end{tabular}

The "widths" of the power burst, $k_{0} E_{T} / \ell P_{\max }$, obtained in the calculations of Table VI, are displayed as open dots in Fig. 3. They are always larger than the widths expected from Bethe-Tait calculations, and (for given $k_{0}^{3} / \ell^{2}$ ) the deviation increases with increasing value of $k_{0}$.

F. Variation of Wave Velocity

In Appendix A we show that, for the simplified equation of state $(\alpha=B=0)$, the square of the velocity of wave propagation is

$$
c^{2}=\left(\frac{\partial p}{\partial \rho}\right)_{s}=\left(-\tau^{\prime}+p\right) \frac{\beta^{\prime}}{A^{\prime}} \rho^{-2}
$$

if the pressure $\mathrm{p}$ is positive and vanishes otherwise. A higher velocity of wave propagation results in a more rapid spreading of the pressure wave and should result in a yield of higher energy. To check this, a number of calculations were performed with $Q^{*}$ and $\beta^{\prime} / A^{\prime}$ constant and varying $\tau^{\prime}$. In these calculations the lifetime was kept short, $l \approx 0.02 \mu$ sec, and 3 cases of reactivity input have been considered.

The results are in Table VII. In each case the energy yield is almost the same for the two lowest values of $-T^{\prime}$. Thereafter it starts increasing at a higher rate. When $\tau^{\prime}=1$, the velocity of wave propagation is $0.53 \mathrm{~cm} / \mu \mathrm{sec}$. Multiplication of the velocity by the length of time from the moment the pressure has started until most of the energy has been generated (as given by IBM-704), we obtained for problems 65, 66, and 67 values of $29 \mathrm{~cm}, 17 \mathrm{~cm}$, and $8 \mathrm{~cm}$, respectively. 
Table VII

VARIATION OF WAVE VELOCITY

\begin{tabular}{|c|l|l|l|l|c|}
\hline $\begin{array}{c}\text { Problem } \\
\text { No. }\end{array}$ & $\begin{array}{c}k_{0}^{3} / \ell^{2} \\
\left(\mu \mathrm{sec}^{-2}\right)\end{array}$ & $\begin{array}{c}-\tau \\
\text { (megabar) }\end{array}$ & $\begin{array}{c}\text { Excess Energy } \\
\text { Yield (10 }\end{array}$ & $\begin{array}{c}\text { Center Peak }) \\
\text { Pressure } \\
(\text { megabar })\end{array}$ & $\begin{array}{c}\text { Maximum } \\
\text { Kinetic } \\
\text { Energy } \\
\left(10^{12} \text { erg }\right)\end{array}$ \\
\hline 59 & $7.003 \times 10^{-9}$ & 0.15 & 853 & 0.0208 & 10.20 \\
56 & $7.003 \times 10^{-9}$ & 0.2682 & 856 & 0.0155 & 5.29 \\
62 & $7.003 \times 10^{-9}$ & 0.5 & 885 & 0.0111 & 2.64 \\
65 & $7.003 \times 10^{-9}$ & 1.0 & 956 & 0.0082 & 1.13 \\
60 & $4.45 \times 10^{-7}$ & 0.15 & 2,378 & 0.113 & 58. \\
57 & $4.45 \times 10^{-7}$ & 0.2682 & 2,380 & 0.101 & 115. \\
63 & $4.45 \times 10^{-7}$ & 0.5 & 2,399 & 0.0846 & 77.3 \\
66 & $4.45 \times 10^{-7}$ & 1.0 & 2,437 & 0.0646 & 38.8 \\
61 & $1.665 \times 10^{-4}$ & 0.15 & 23,752 & 1.391 & $8,681$. \\
58 & $1.665 \times 10^{-4}$ & 0.2682 & 23,744 & 1.374 & $8,621$. \\
64 & $1.665 \times 10^{-4}$ & 0.5 & 23,919 & 1.357 & $5,343$. \\
67 & $1.665 \times 10^{-4}$ & 1.0 & 24,183 & 1.313 & $4,802$. \\
\hline
\end{tabular}

A significant increase in energy yield, however, is realized only in problem 65, in which "the distance the wave has traveled through" is larger than the radius of the core. We notice also that in all the cases pressure is a more sensitive function of $\tau^{\prime}$ than is the energy yield. This can be understood if it is realized that the propagation of the pressure wave at constant velocity does not change $\int p d V$ and thus affects $\ddot{k}$, given by Eq. (8), only very little and causes only small increase in the energy yield. In Fig. 9 we have displayed the center peak pressure as a function of initial wave velocity,

$$
c_{0}^{2}=\tau^{\prime} \beta^{\prime} v_{0}^{2} / A^{\prime}
$$

The decrease of the kinetic energy realized with increasing $-\tau^{\prime}$ looks even larger than the decrease in pressure. Thus it is likely that the destructive potential of the burst will decrease with increasing $-\tau^{\prime}$ in spite of increasing energy yield.

\section{G. Kinetic Energy}

In all the tables of this report we have given also the maximum kinetic energy obtained during the running of the problem on the IBM-704. The kinetic energy at the end of the run has always been close to this value. The cases for which kinetic energy has been rising at an appreciable rate at the end of the run have been indicated with asterisks. During the run, kinetic energy keeps increasing when energy is being generated at an appreciable rate. It increases later, at a somewhat lower rate, when 
the pressure wave is reflected from a rarer medium and decreases somewhat when the pressure wave is reflected from a denser medium. At other times the total kinetic energy is a roughly constant value. The value of kinetic energy given here is not always the maximum value obtainable from the excursion, since in most cases at the end of the run the pressure has not yet vanished every place. Thus continuation of the run would very likely result in an increase in the kinetic energy.

In a few cases, the maximum kinetic energy that could be obtained by an isentropic expansion has been computed. We have taken Eq. (16a) as the maximum energy available per gram of uranium and summed over the core, using our standard parabolic shape for power and energy distribution. The result of this calculation has always given a much larger value for kinetic energy than obtained at the end of the IBM-704 run. Sometimes the kinetic energy has been only $\frac{1}{10}$ of what one expects to get by an isentropic expansion. Thus it seems probable that in all these cases a large amount of potential kinetic energy has been used up by heating the core and the blanket.

So, while our calculations indicate that the composition of the blanket has only a small effect upon the total energy yield, it may have a large influence on the amount of kinetic energy eventually realized. In like manner. only small changes of specific volume are realized during appreciable energy generation. But an equation of state valid for large changes of volume is needed to compute the kinetic energy. Since our simplified equation of state is very unlikely to be adequate in an actual situation, kinetic energies obtained here have only a qualitative theoretical significance. 
32

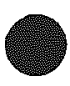

. 
Appendix A

ON THE EQUATIONS OF STATE USED IN AX-I

The AX-I program uses both thermal and caloric equations of state. In this code, they have the simple linear forms

$$
\begin{aligned}
p & =\tau+\beta \theta+\alpha \rho \\
c_{V} & =\left(\frac{\partial e}{\partial \theta}\right)_{\rho}=A+B \theta
\end{aligned}
$$

where $p$ is pressure, $c_{\mathrm{y}}$ is the specific heat at constant volume, $\theta$ is the absolute temperature, $\rho$ is the density, and $\tau, \beta, \alpha, A$, and $B$ are input parameters for a given problem. During a fast excursion, while heat is being generated, we can reasonably assume that there is no heat conduction. Thus information about temperature $\theta$ is superfluous. All we need for an adiabatic calculation is the relationship among pressure $p$, density $\rho$, and internal energy $e$. This gives us only one equation of state. Indeed, it is quite thinkable to have two substances that have the same relationship among $p, \rho$, and $e$ and differ in absolute temperature $\theta$. Those two substances would behave identically in our calculations. Thus it is of interest, starting from Eqs. (1a) and (2a), to obtain the relationship among $p, p$, and e.

Integrating Eq. (2a), we obtain

$$
e=A \theta+\frac{1}{2} B \theta^{2}+f(\rho) \text {, }
$$

where $f(\rho)$ is a yet undetermined function that has to be such that the second law of thermodynamics is satisfied. The second law says

$$
\frac{1}{\theta}\left(\mathrm{de}-\mathrm{p} \frac{\mathrm{d} \rho}{\rho^{2}}\right)=\mathrm{ds} \quad \text { : }
$$

or, after substitution of Eqs. (1a) and (3a),

$$
\frac{1}{\theta}\left[(A+B \theta) d \theta+\left(f^{\prime}(\rho)-\frac{\tau+\beta \theta+\alpha \rho}{\rho^{2}}\right) d \rho\right]=d s \text {. }
$$

This is a complete differential; thus,

$$
\frac{\partial}{\partial \rho}\left(\frac{A+B \theta}{\theta}\right)=\frac{\partial}{\partial \theta} \frac{1}{\theta}\left[f^{\prime}(\rho)-\frac{\tau+\beta \theta+\alpha \rho}{\rho^{2}}\right]
$$


or

$$
0=-\frac{1}{\theta^{2}}\left[f^{\prime}(\rho)-\frac{\tau+\alpha \rho}{\rho^{2}}\right] \text {. }
$$

Integrating this expression, we find that

$$
f(\rho)=-\frac{\tau}{\rho}+\alpha \ln \rho+\text { const. }
$$

Substituting this into Eq. (4a), we obtain

$$
s=A \ln \theta+B \theta+(\beta / \rho)+\text { const. }
$$

Similarly, from Eq. (3a) we have

$$
e=A \theta+\frac{1}{2} B \theta^{2}-(\tau / \rho)+\alpha \ln \rho+\text { const. }
$$

Now, solving for $\theta$ from Eq. (1a) and substituting this value into the preceding expression, we obtain

$$
e=\frac{A}{\beta}(p-\tau-\alpha \rho)+\frac{B}{2 \beta^{2}}(p-\tau-\alpha \rho)^{2}-\frac{\tau}{\rho}+\alpha \ln \rho+\text { const. }
$$

which is the desired relationship among $e, p$, and $\rho$.

This is a rather complex equation even for a constant $\rho$. But we know how to solve the problem of an excursion, neglecting the variation of density for any relationship between $p$ and e, using the generalized BetheTait procedure. Thus in order to investigate the influence of pressure variation with density in later calculations, we have simplified the relationship between $p$ and $e$ by taking $B=0$. Then the relationship between pressure and internal energy becomes linear, like that used by Bethe and Tait. (1) Equation (6a) then simplifies into

$$
\mathrm{e}=\frac{\mathrm{A}}{\beta} \mathrm{p}-\frac{T}{\rho}+\alpha \ln \rho-\alpha \frac{\mathrm{A}}{\beta} \rho+\text { const. }
$$

Here the variation of internal energy with density is still quite complex. However, since in the perturbation treatment only small (linear) variations of density are considered, we are interested only in

$$
\left(\frac{\partial e}{\partial \rho}\right)_{p}=\frac{\tau}{\rho^{2}}+\frac{\alpha}{\rho}-\alpha \frac{A}{\beta}
$$

evaluated at the initial density. Thus, within first-order perturbation theory we should obtain identical results with any two sets of constants 
if $A / \beta$ and $(\partial \mathrm{e} / \partial \rho)_{\mathrm{p}}$ are the same in the two sets. If these conditions are satisfied, one constant is superfluous. Thus in our later calculations we have chosen

$$
\alpha^{\prime}=0
$$

(where the prime denotes a new set of constants) and

$$
\left(\frac{\partial \mathrm{e}}{\partial \rho}\right)_{\mathrm{p}}=\frac{\tau^{\prime}}{\rho^{2}}=\frac{\tau^{\prime}}{\rho^{2}}+\frac{\alpha}{\rho}-\alpha \frac{\mathrm{A}}{\beta} .
$$

Our simplified equations of state then read

$$
\begin{aligned}
\mathrm{p} & =\tau^{\prime}+\beta^{\prime} \theta \\
c_{\mathrm{V}} & =A^{\prime}
\end{aligned}
$$

and the relationship among pressure, internal energy, and specific volume $\mathrm{v}=1 / \rho$, is then

$$
p=\left(\beta^{\prime} / A^{\prime}\right)\left(e+\tau^{\prime} v\right)+\text { const }
$$

The integration constant is eliminated by measuring energy density e from the initial conditions, when $\theta=\theta_{i}$ and $v=v_{0}$ :

$$
p=\frac{\beta^{\prime}}{A^{\prime}}\left[e-A^{\prime}\left(\frac{-\tau^{\prime}}{\beta^{\prime}}-\theta_{i}\right)\right]+\frac{\beta^{\prime}}{A^{\prime}} \tau^{\prime}\left(v-v_{0}\right) \text {. }
$$

To check our contention that only a linear variation of pressure with specific volume is of importance, we have chosen as a basis 3 excursions: problems 30,31 , and 32 with constants for the equation of state as given in Section III-D. Then we have determined a new set of constants so that Eq. (7a) and Eq. (8a) are satisfied and the rate of change of pressure with energy density is the same:

$$
\beta^{\prime} / A^{\prime}=\beta / A,
$$

as well as the threshold energy density

$$
\begin{aligned}
Q^{*} & =A^{\prime}\left(\theta^{\prime} \mathrm{sp}-\theta_{i}\right)=A\left(\theta_{\mathrm{sp}}-\theta_{i}\right) \\
& =A^{\prime}\left(-\frac{\tau^{\prime}}{\beta^{\prime}}-\theta_{i}\right)=A\left(-\frac{\tau+\alpha \rho}{\beta}-\theta_{i}\right)
\end{aligned}
$$

The calculations with the new set of constants:

$$
\begin{aligned}
& \alpha^{\prime}=0 ; \quad \beta^{\prime}=1290.1 \mathrm{~g} / \mathrm{cm}-\mu \mathrm{sec}^{2}-\mathrm{kev} ; \\
& T^{\prime}=-0.2682 \mathrm{~g} / \mathrm{cm}-\mu \mathrm{sec}^{2} ; \quad A^{\prime}=72.42 \mathrm{~cm}^{2} / \mu \mathrm{sec}^{2}-\mathrm{kev}, \quad B=0,
\end{aligned}
$$


are reported in Table VIII. Agreement is quite satisfactory, even for a sensitive quantity, center peak pressure.

Table VIII

LINEARIZATION OF PRESSURE DEPENDENCE ON DENSITY

\begin{tabular}{|c|c|c|c|c|}
\hline $\begin{array}{c}\text { Problem } \\
\text { No. }\end{array}$ & $\begin{array}{c}\text { Excess } \\
\left(\mu \mathrm{sec}^{3} / \mathrm{l}^{2}\right.\end{array}$ & $\begin{array}{c}\text { Center } \\
\text { Energy } \\
\text { Yield } \\
\left(10^{12} \mathrm{erg}\right)\end{array}$ & $\begin{array}{c}\text { Maximum } \\
\text { Pressure } \\
(\text { megabar })\end{array}$ & $\begin{array}{c}\text { Kinetic } \\
\text { Energy } \\
\left(10^{12} \mathrm{erg}\right)\end{array}$ \\
\hline 30 & $7.003 \times 10^{-9}$ & 870 & 0.0156 & 5.65 \\
31 & $4.45 \times 10^{-7}$ & 2,380 & 0.1008 & 132. \\
32 & $1.665 \times 10^{-4}$ & 23,840 & 0.376 & 8584. \\
56 & $7.003 \times 10^{-9}$ & 856 & 0.0155 & 5.29 \\
57 & $4.45 \times 10^{-7}$ & 2,380 & 0.101 & 115. \\
58 & $1.665 \times 10^{-4}$ & 23,744 & 1.374 & 8621. \\
\hline
\end{tabular}

For materials of nearly normal density, constants for the present equations of state can be simply estimated from experimental measurements. Usually, measurements of specific heat are available. Also, the cubical coefficient of thermal expansion,

$$
\frac{1}{\rho} \frac{\mathrm{d} \rho}{\mathrm{d} \theta}=\frac{\beta}{\alpha \rho}
$$

is known as well as the velocity of propagation of sound, c. By means of Eqs. (1a), (5a), and (2a), the velocity of sound can easily be expressed in terms of our parameters:

$$
\begin{aligned}
c^{2} & =\left(\frac{\partial p}{\partial \rho}\right)_{s}=\alpha+\beta\left(\frac{\partial \theta}{\partial \rho}\right)_{s} \\
& =\alpha+\frac{\beta^{2} \theta}{\rho^{2}(A+B \theta)} \\
& =\alpha+\frac{\beta^{2} \theta}{\rho^{2} c_{V}}
\end{aligned}
$$

where usually the second term is small and the square of the wave velocity is approximately equal to $\alpha$.

Of course for our simplified equation of state this is no longer true. When $\alpha$ and $B$ vanish, the preceding equation and Eq. (9a) yield 


$$
c^{2}=\frac{\beta^{2} \theta}{\rho^{2} A^{\prime}}=\frac{\beta^{1}}{A^{1}} v^{2}\left(-\tau^{\prime}+p\right)
$$

Thus the velocity of wave propagation increases as $p$ increases and experiences a sudden jump when pressure goes from vanishing to small positive as voids are being eliminated.

After the heat generation is over, the core material expands, presumably doing some work and imparting some kinetic energy to the reactor material. The maximum work that a core element can produce is obtained on the assumption that the expansion is isentropic. For the simplified equation of state $(\alpha=B=0)$, taking Eq. (5a) and substituting $\theta$ from Eq. (9a), we see that during an isentropic expansion

$$
A^{\prime} \ln \frac{p-\tau^{\prime}}{\beta^{\prime}}+\beta^{\prime} v=\text { const. }
$$

Supposing that the expansion continues until the pressure vanishes. Substituting $v$ from Eq. (1la), we see that the energy lost and the work done is

$$
w=\frac{A^{\prime} \tau^{\prime}}{\beta^{\prime}}\left(\ln \frac{p_{1}-\tau^{\prime}}{-\tau^{\prime}}+\frac{p_{1}}{\tau^{\prime}}\right),
$$

where subscript 1 refers to the state preceding expansion. Now, assuming that the initial volume is equal to $v_{0}$ and calling the energy density above threshold

$$
e_{e x}=e-A^{\prime}\left(\frac{-\tau^{\prime}}{\beta^{\prime}}-\theta_{i}\right),
$$

we see from Eq. (11a) that the pressure is proportional to the excess energy density, ex. Thus, Eq. (15a) can be rewritten

$$
\frac{w}{e_{e x}}=1-\frac{-\tau^{\prime} A^{\prime}}{\beta^{\prime} e_{e x}} \ln \left(1+\frac{\beta^{\prime} e_{e x}}{-\tau^{\prime} A^{\prime}}\right) \text {. }
$$

So the energy available to do work usually is considerably smaller than energy generated over threshold. If $\beta^{\prime} e_{e x} /\left(-\tau^{\prime} A^{\prime}\right)<<1$,

$$
\frac{w}{e_{e x}}=\frac{1}{2}\left(\frac{\beta^{\prime} e_{e x}}{-\tau^{\prime} A^{\prime}}\right)\left[1-\frac{2}{3}\left(\frac{\beta^{\prime} e_{e x}}{-\tau^{\prime} A^{l}}\right)+\ldots\right]
$$


Appendix B

\section{CONNECTION BETWEEN NUCLEAR PARAMETERS \\ $k_{0}, \ell$. AND THE EQUATION OF STATE}

First-order perturbation treatment yields an energy reduction proportional to the displacement, Eq. (6):

$$
k-k_{0}=\int \rho u \cdot f d v
$$

When the equation of motion, Eq. (2),

$$
\ddot{\mathrm{u}}=-\frac{1}{\rho} \nabla \mathrm{p} \approx-\frac{1}{\rho_{0}} \nabla \mathrm{p},
$$

is used, the mechanism of reactivity reduction can be described by

$$
\ddot{k}=\int p \nabla \cdot f d V .
$$

If delayed neutrons are neglected, the reactivity is connected with the energy input by Eq. (19):

$$
k=\ell \frac{\mathrm{d}}{\mathrm{d} t} \ln \dot{Q}
$$

The shape of the power is known and so are the equations of state. If one uses them for calculation of the pressure, neglecting expansion and wave propagation, one can express $p$ in Eq. (3b) in terms of energy input. Then Eq. (3b) and Eq. (4b) become coupled ordinary differential equations describing the excursion in the Bethe-Tait approximation.

If a step input of reactivity is to be considered and the excursion is assumed to start with negligible power, initial conditions for these differential equations are

$$
Q \approx 0 ; \quad \dot{Q}=\left(k_{0} / l\right) Q ; \quad k=k_{0} ; \quad \dot{k}=0
$$

We can eliminate these initial conditions formally by measuring time in terms of initial period, $l / k_{0}$, and the excess reactivity in terms of $k_{0}$. In terms of these dimensionless quantities, Eq. (4b) can be rewritten as

$$
\left(\frac{\mathrm{k}}{\mathrm{k}_{0}}\right)=\left(\frac{\mathrm{l}}{\mathrm{k}_{0}} \frac{\mathrm{d}}{\mathrm{dt}}\right) \ln \dot{\mathrm{Q}}
$$

which is free of any parameters. If, in addition, we measure length in terms of $b_{0}, E q .(1 b),(2 b)$, and (3b) can be rewritten in dimensionless form: 


$$
\begin{aligned}
& \left(\frac{k}{k_{0}}\right)-1=\int \rho\left(\frac{u}{b k_{0}}\right) f b d V, \\
& \left(\frac{\ell}{k_{0}} \frac{d}{d t}\right)^{2}\left(\frac{u}{b k_{0}}\right) \approx-\frac{b}{\rho_{0}} \nabla\left(\frac{\ell^{2}}{b^{2} k_{0}^{3}} p\right),
\end{aligned}
$$

and

$$
\left(\frac{l}{k_{0}} \frac{d}{d t}\right)^{2}\left(\frac{k}{k_{0}}\right)=\int\left(\frac{l^{2}}{b^{2} k_{0}{ }^{3}} p\right) \nabla \cdot f b^{2} d V
$$

Our simplified equation of state, Eq. (11a), can also be rewritten as

$$
\begin{aligned}
\left(\frac{\mathrm{p} v_{0} l^{2}}{\mathrm{~b}^{2} \mathrm{k}_{0}{ }^{3}}\right)= & {\left[\frac{\beta^{\prime}}{\mathrm{A}^{\prime}} \mathrm{v}_{0} \mathrm{k}_{0}\right]\left(\mathrm{e} \frac{\ell^{2}}{\mathrm{~b}^{2} \mathrm{k}_{0}^{4}}\right)-\left[\left(-\tau^{\prime}-\beta^{\prime} \theta_{i}\right) \mathrm{v}_{0} \frac{\ell^{2}}{\mathrm{~b}^{2} \mathrm{k}_{0}^{3}}\right] } \\
& +\left[\frac{\beta^{\prime}}{\mathrm{A}^{\prime}} \mathrm{v}_{0} \mathrm{k}_{0}\right]\left[\tau^{\prime} \frac{\ell^{2}}{\mathrm{~b}^{2} \mathrm{k}_{0}^{3}} \mathrm{v}_{0}\right]\left(\frac{\mathrm{v}-\mathrm{v}_{0}}{\mathrm{v}_{0} \mathrm{k}_{0}}\right),
\end{aligned}
$$

where the variables in parentheses and the constants in square brackets are all dimensionless. In addition, we can also take a constant factor out of $\nabla \cdot f$ and include it in the definitions of dimensionless $u$ and $p$. Thus, the progress of the excursion, described by these dimensionless variables, depends upon three dimensionless parameters in the equation of state. And the variation of the parameters $k_{0}$ and $k_{0}{ }^{3} / \ell^{2}$ we have performed in Chapter III is equivalent to the variation of $\beta^{\prime} / A^{\prime}$ and $\left(\beta^{\prime} / A^{\prime}\right) Q^{*}=$ $\left(-\tau^{\prime}-\beta \theta_{i}\right)$, which have been kept constant. To check this contention, we have chosen problems 65, 66, 67, 72, and 75 as references. Then we have reduced $\mathrm{k}_{0}$ by 2 , kept $\ell$ the same, and simultaneously have changed the constants of equation of state, Eq. (11a), to keep the parameters in the square brackets in Eq. (5b) the same ( $\theta_{i}$ has also been kept the same). Then, as seen from Eq. (5b), one expects to obtain only $\frac{1}{16}$ of previous energy yield and $\frac{1}{8}$ of previous pressure. The results of these calculations are in Table IX. The discrepancy between expected and actually obtained values is noticeable in the last three problems. However, at least a part of it can be explained by failure of the synthetic viscous pressure, given by Eq. (23), to conform with our scaling and by other inaccuracies of the numerical procedure. Thus the agreement seems satisfactory. We have plotted the yields of excursions in Fig. 6 in dimensionless notation as solid dots. The dimensionless excess energy $E_{e x} / E^{*}$ has been plotted in Fig. 10 , and the dimensionless center peak pressure $p_{\max } /\left(-\tau-\beta^{\prime} \theta_{i}\right)$ in Fig. 11. As arguments we have used $x$, of Eq. (32), and

$$
y=\left[\frac{5}{32} \frac{\Sigma_{t r} \nu \Sigma_{f}\left[1-(6 q / 5)+\left(3 q^{2} / 7\right)\right] b^{2}}{F \sqrt{q}}\right]\left(\frac{\beta^{\prime} v_{0}}{A^{\prime}}\right) k_{0} .
$$


The third dimensionless parameter, from the equation of state, Eq. (5b), $\tau^{\prime} /\left(\tau^{\prime}+\beta^{\prime} \theta_{i}\right)$, was equal to 1.0776 for the whole set. In addition, we have performed 3 calculations with this third parameter equal to 1.2 and 3 calculations with parameter value equal to 1.0447 . In these 6 calculations the threshold energy density was chosen higher: $Q^{*}=0.012546$, but $\beta^{\prime} / \mathrm{A}^{\prime}$ and the $y^{\prime} s$ have been the same as in the reference problems of Table VI. These calculations have been indicated by open dots and should lie on solid lines of Figs. 10 and 11 . Two open dots indicating low-pressure yield in Fig. 11 demonstrate that the yield is sensitive to the third parameter, $\tau^{i} /\left(\tau^{\prime}+\beta^{\prime} \theta_{i}\right)$, if the excursion is small.

Table IX

CONNECTION BETWEEN NUCLEAR AND EQUATION OF STATE PARAMETERS

\begin{tabular}{|c|c|c|c|c|c|}
\hline $\begin{array}{c}\text { Problem } \\
\text { No. }\end{array}$ & $\begin{array}{c}\text { Excess Energy } \\
\text { Yleld } \\
\left(10^{12} \text { erg) }\right.\end{array}$ & $\begin{array}{c}\text { Center } \\
\text { Peak Pressure } \\
\text { (megabar) }\end{array}$ & $\begin{array}{c}\text { Problem } \\
\text { No. }\end{array}$ & $\begin{array}{c}\text { Excess Energy } \\
\text { Y1eld } \\
\left(10^{12} \text { erg }\right)\end{array}$ & $\begin{array}{c}\text { Center } \\
\text { Peak Pressure } \\
\text { (megabar) }\end{array}$ \\
\hline 65 & 956 & 0.0082 & 305 & 59 & 0.00104 \\
66 & 2,437 & 0.0646 & 266 & 151 & 0.0081 \\
67 & 24,183 & 1.313 & 267 & 1,488 & 0.164 \\
72 & 1,169 & 0.008 & 272 & 195 & 0.0018 \\
75 & 3,014 & 0.06 & 275 & 0.0098 \\
\hline
\end{tabular}

Though Figs. 10 and 11 have been plotted in dimensionless form, the calculations have been done for the same power shape and assuming the same material in the blanket as in the core. We already know that the power shape changes the yield, even in the simple Bethe-Tait calculation (see Fig. 1 and Table I). In addition, the change of material in the blarket may be expected to induce larger variation in the yield than obtained in Section III-D. Calculations in that section were performed for very short lifetimes and served mainly to demonstrate the lack of influence of blanket material in the Bethe-Tait approximation.

As we have seen, when the Bethe-Tait method is applicable, expansion during an excursion is negligible as well as wave propagation. Then only the relationship between pressure and energy at the initial density of the core is needed to determine the yield. In addition, two relationships between pressure and energy that differ only in a constant scale factor for energy produce identical pressures and reactivities during the burst. Thus we have seen for simple Eq. (16) that progress of the excursion was described only by $(\gamma-1) Q^{*}$ and did not involve $(\gamma-1)$ by itself. 
It is instructive to consider a simple equation of state without a threshold:

$$
p=A Q^{n}, \text { for } \rho=\rho_{C}
$$

Then, since progress of the excursion does not depend on a scaling factor of power input, pressure and reactivity in the Bethe-Tait approximation do not depend on $A$. In particular, our dimensionless pressure average over the core,

$$
\frac{\overline{\mathrm{p}} l^{2}}{\rho_{\mathrm{c}} \mathrm{b}^{2} \mathrm{k}_{0}^{3}}\left(-\nabla \cdot \mathrm{f} \frac{4 \pi}{3} \mathrm{~b}^{3} \rho_{\mathrm{c}} \mathrm{b}^{2}\right) \approx \frac{\overline{\mathrm{p}} \rho^{2}}{\rho_{\mathrm{c}} \mathrm{b}^{2} \mathrm{k}_{0}^{3}}\left(\frac{16 \mathrm{q}^{2} \mathrm{~F}}{\sum_{t \mathrm{r}} \nu \sum_{\mathrm{f}} \mathrm{b}^{2}\left[1-(6 \mathrm{q} / 5)+\left(3 \mathrm{q}^{2} / 7\right)\right]}\right) \text {. }
$$

is a function of dimensionless time and $\mathrm{n}$ only. We have computed the maximum of Eq. (8b) by the Runge-Kutta method on the LGP-30 and displayed the results in Table III. As demonstrated in the comparisons of Figs. 10 and 11 , however, by means of the Bethe-Tait method one expects to obtain more accurate values for the energy yield than for the peak pressure. Thus, the peak pressure obtained here should be regarded only as an intermediate step to estimate the energy yield from Eq. (7b). 


\section{REFERENCES}

1. Bethe, H. A., and Tait, J.H., An Estimate of the Order of Magnitude of the Explosion When the Core of a Fast Reactor Collapses, UKAEARHM (56)/113.

2. McCarthy, W. J., et al., Studies of Nuclear Accidents in Fast Power Reactors, Proceedings of the Second United Nations International Con ference on the Peaceful Uses of Atomic Energy, Geneva, Switzerland, $12,207(1958)$.

3. Okrent, D., et al., AX-I, A Computing Program for Coupled NeutronicsHydrodynamics, Calculations on the IBM-704, ANL-5977.

4. Nicholson, R. D., APDA (Private Communication).

5. Schmitt, A. P., Saclay, France (Private Communication).

6. Koch, L.J., et al. Hazards Summary Report - Experimental Breeder Reactor II (EBR-II), ANL-5719.

7. Von Neumann, J., and Richtmyer, R. D., J. Applied Phys. 21, 232 (1950).

8. Stratton, W.R. (Private Communication).

9. Stratton, W. R。, et al. Analysis of Prompt Excursions in Simple Systems and Idealized Fast Reactors, Proceedings of the Second United Nations International Conference on the Peaceful Uses of Atomic Energy, Geneva, Switzerland, 12, 196 (1958).

10. Reactor Physics Constants, ANL-5800, Revised Edition, Section 5.5.1.

11. Bethe, H. A. (Private Communication).

12. Jankus, V.Z., A Parameter Study Using AX-I, Trans. Amer。Nuc. Soc. 2. 115 (June 1959).

13. Lehman, J.P., Hatchet - A Coupled Neutronics-Hydrodynamics Code to Calculate Burst Characteristics of a Pulsed Reactor for IBM-704, AN-237. 


\section{ACKNOWLEDGMENTS}

The author is grateful to Dr. D. Okrent for his interest in this work, his advice, and encouragement. He is also pleased to acknowledge the generous assistance of $\mathrm{H}$. J. Wheeler, L. B. Miller, L. C. Kvitek, and D. M. O'Shea, whose work preparing numerical programs, running, and processing problems and performing numerical calculations have made this report largely possible. Finally he is indebted to Dr. G. J. Fischer, whose numerous suggestions have helped to improve the readability of this report. 


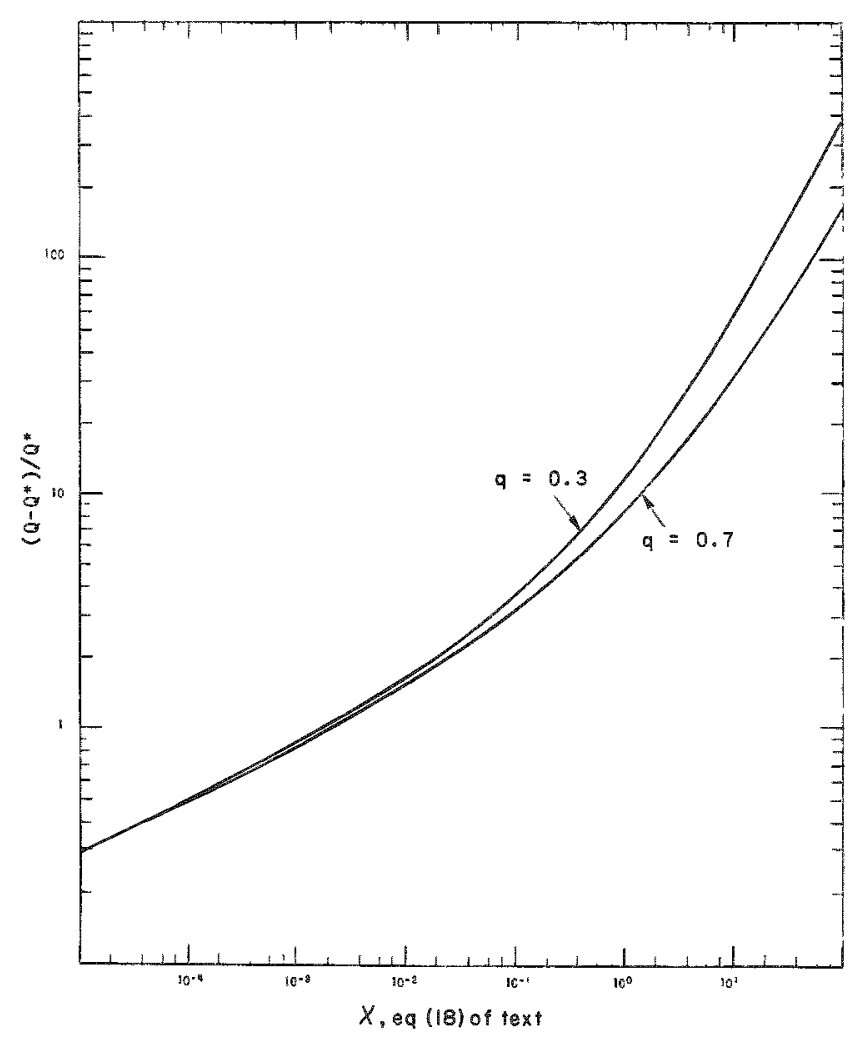

Fig. 1

Ratio of Excess Energy to Threshold Energy in Simple Bethe-Tait Calculation

$112-824$

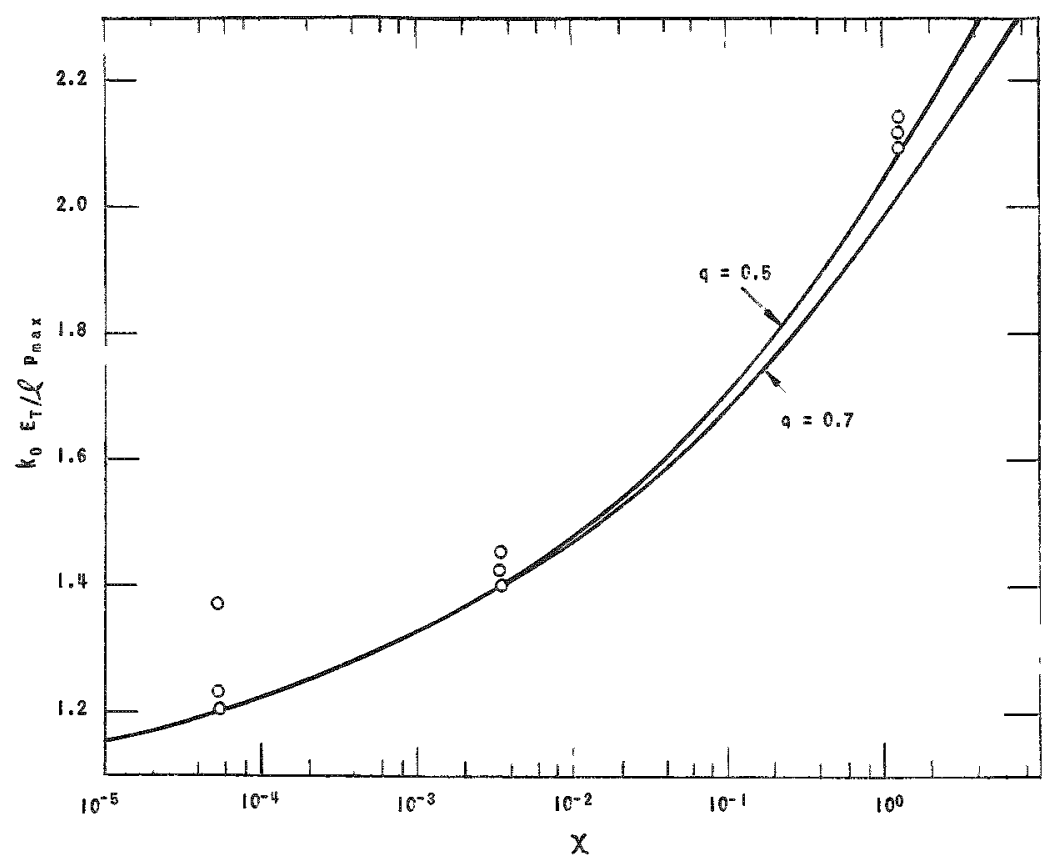

Fig. 2. Width of the Power Burst in Terms of Initial Period $112-820$ 


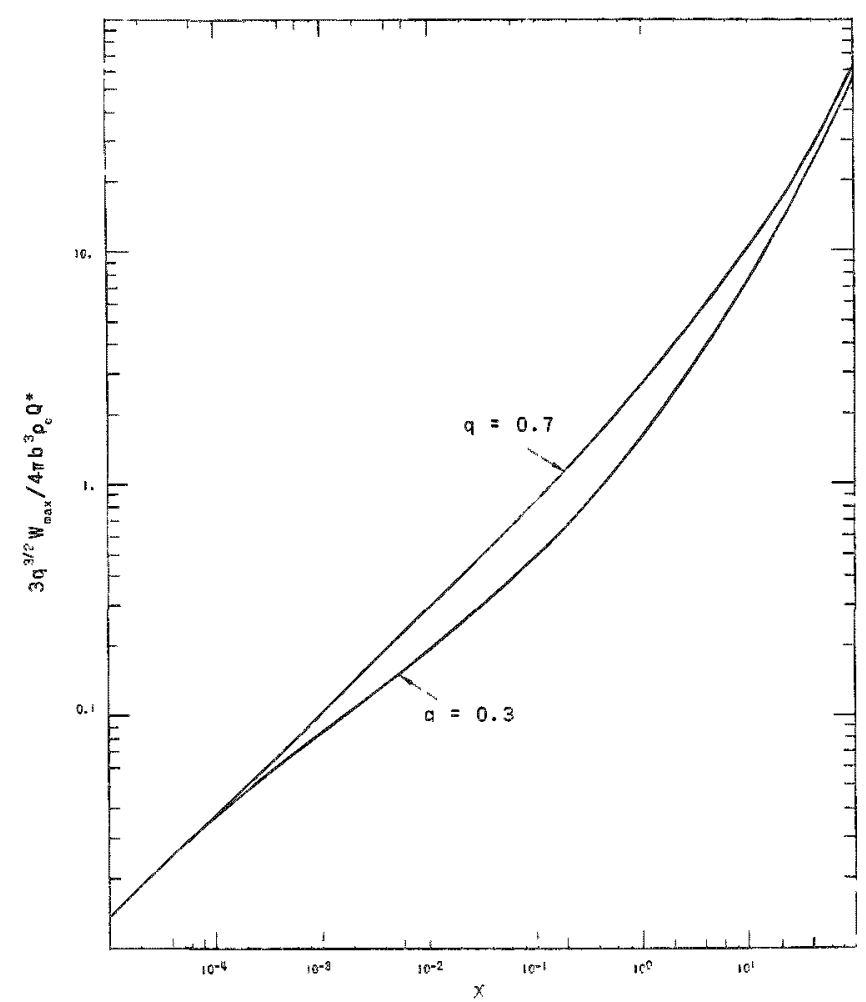

Fig. 3. An Upper Bound for Maximum Work as Given by Eq. (21) $112-825$

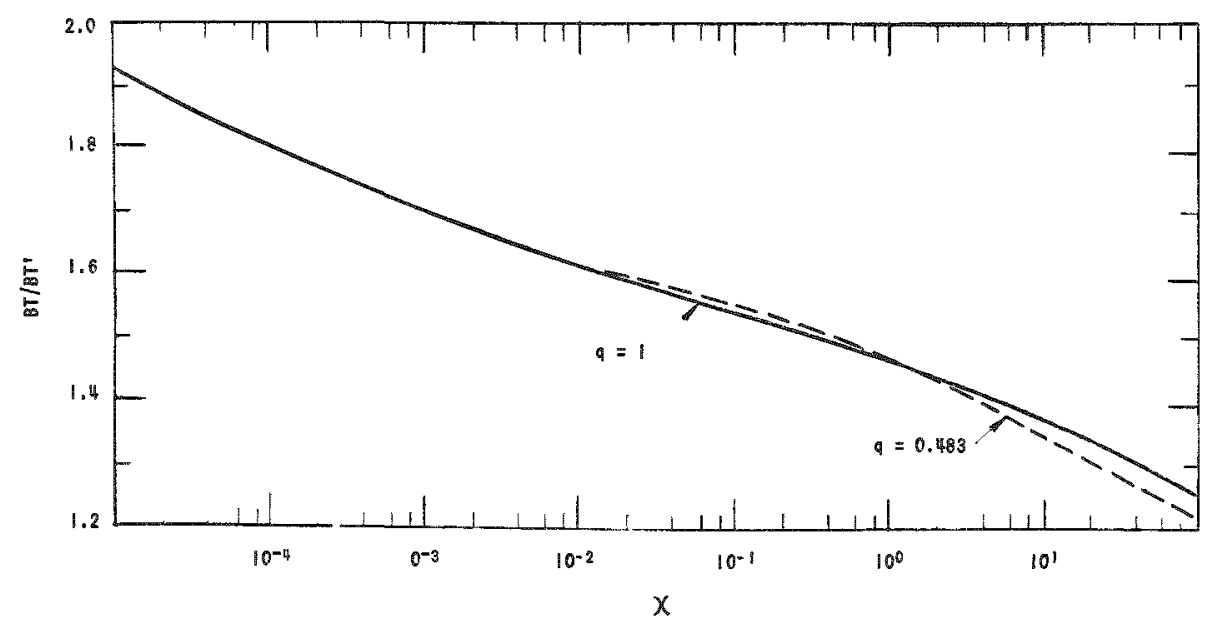

Fig. 4. Ratio of Excess Energy in BT to that in Approximation BT $112-821$ 


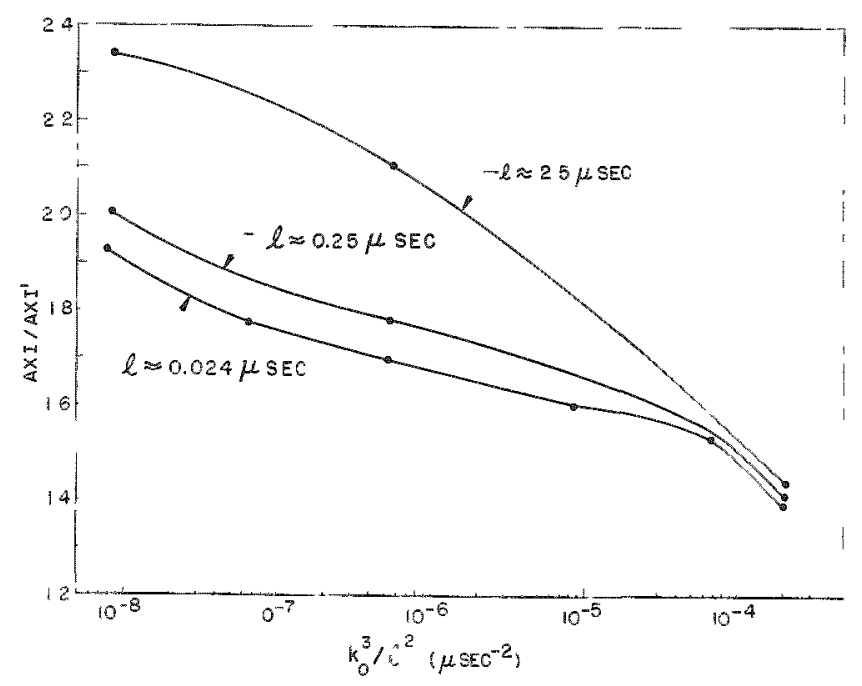

Fig. 5

Ratio of Excess Energy in AX-I to that in $A X-I^{\prime} ; \mathrm{x} \approx 1.10 \times 10^{4}\left(k_{0}^{3} / \ell^{2}\right)$ $111-7829-\mathrm{A}$

Fig. 6

Energy Yield in Excess of Threshold for Various Lifetimes; $E^{*}=2,100 \times 10^{12} \mathrm{ergs}$ $111-7828-A$
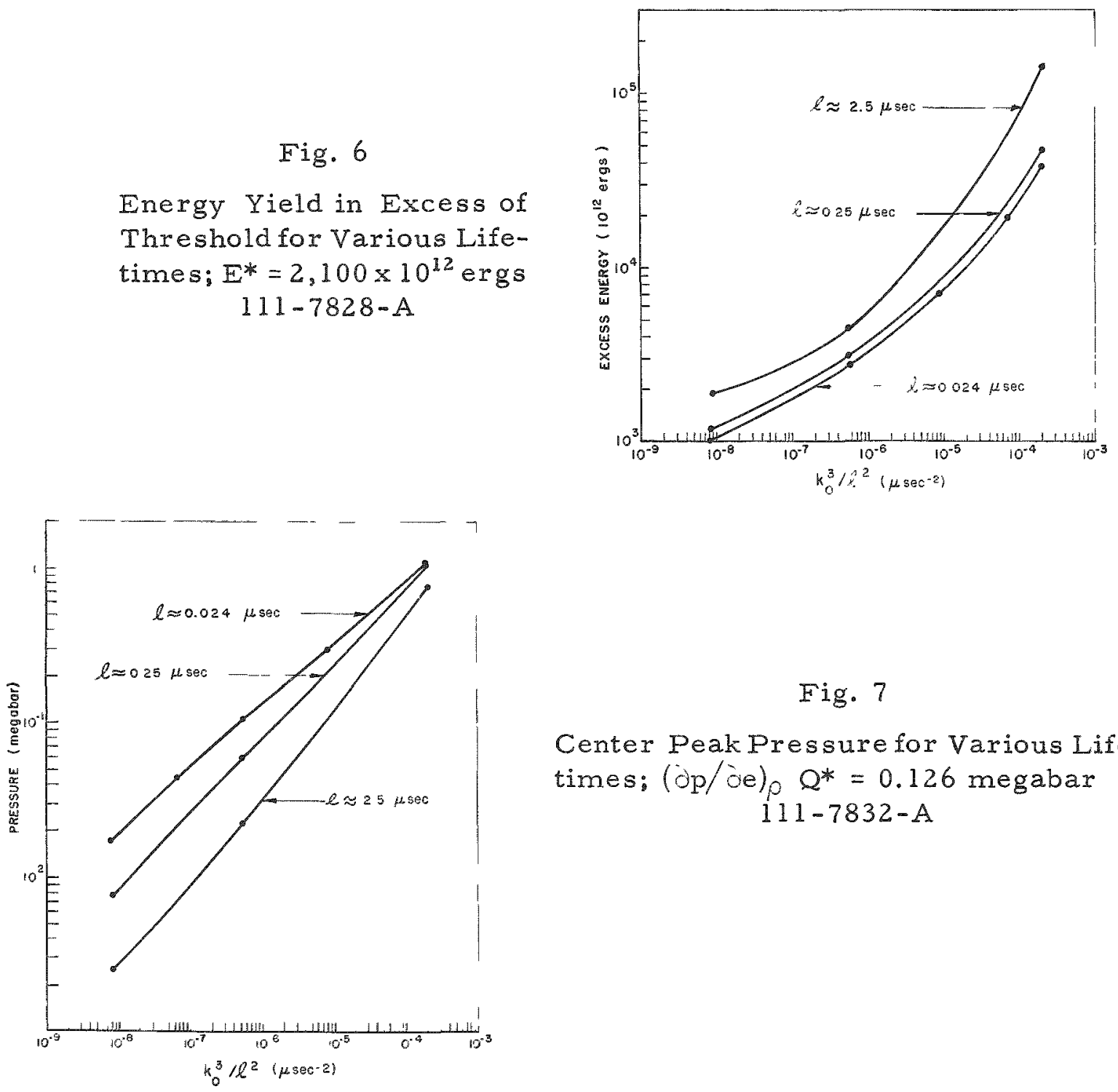

Fig. 7

Center Peak Pressure for Various Lifetimes; $(\mathrm{d} / \mathrm{\partial e})_{\rho} Q^{*}=0.126$ megabar $111-7832-\mathrm{A}$ 


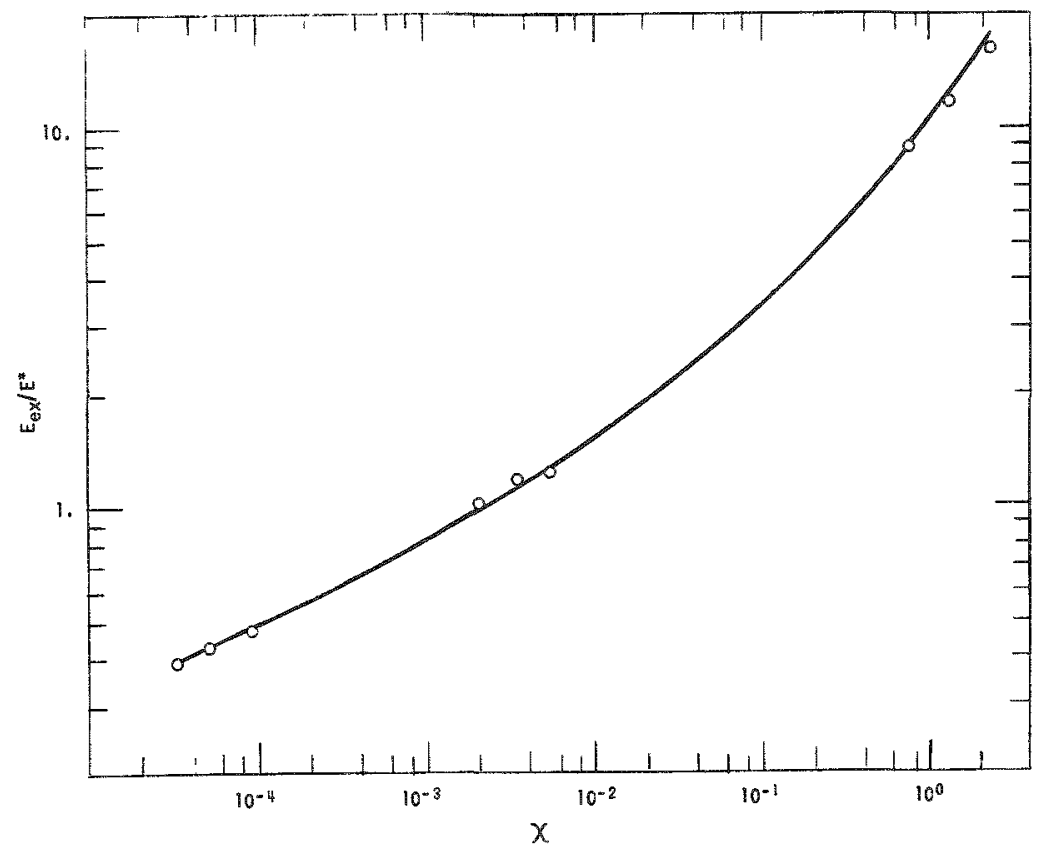

Fig. 8. Effect of Blanket Density $112-818$

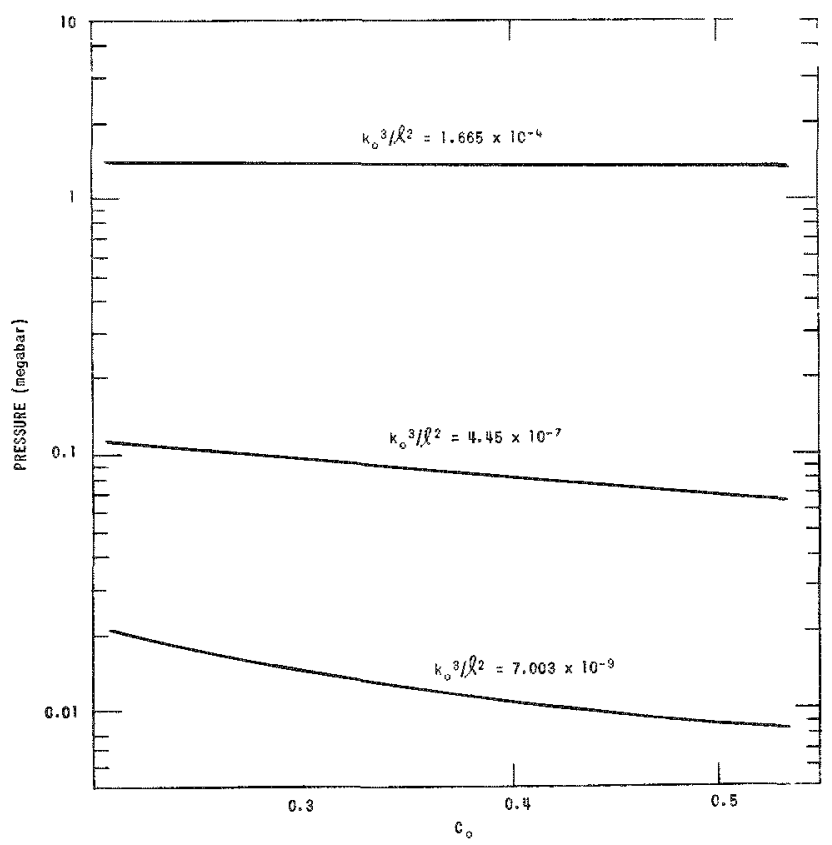

Fig. 9. Variation of Center Peak Pressure with Wave Velocity $112-823$ 


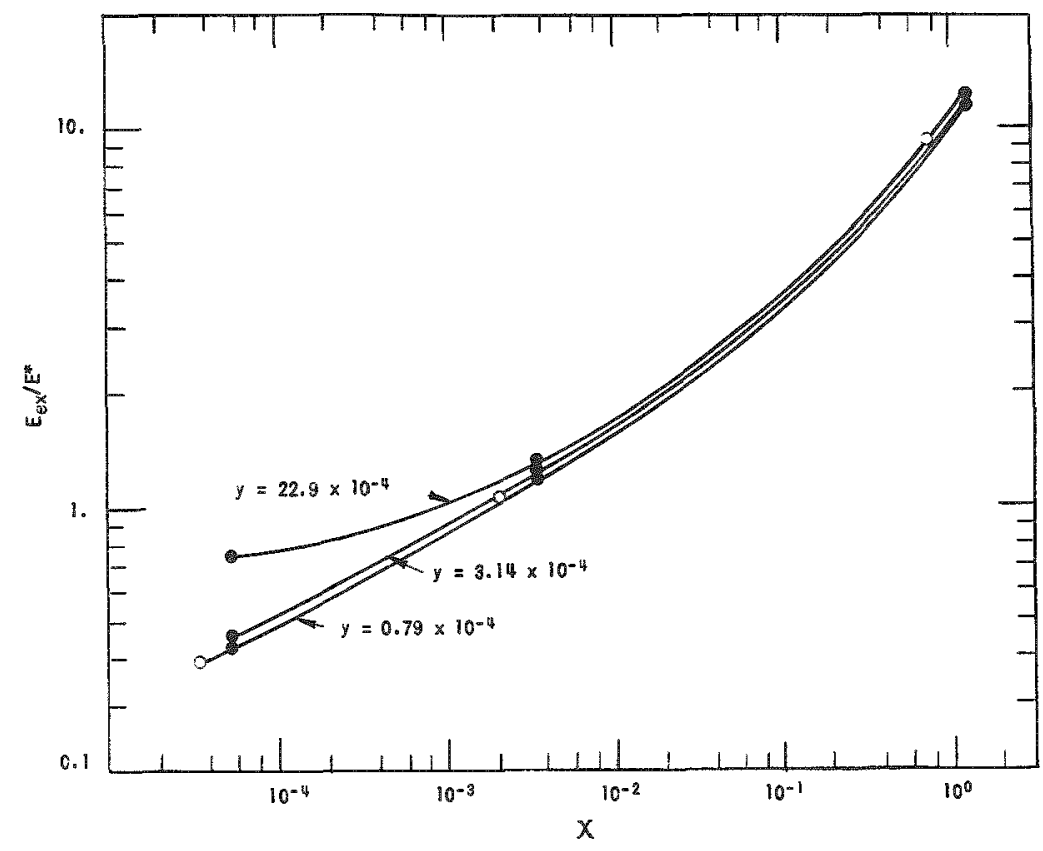

Fig. 10. Dimensionless Excess Energy Yield, Eex/E*, as a Function of Dimensionless Parameters $x$ Eq. (32) and y Eq. $(6 \mathrm{~b}) ; \tau^{\prime} /\left(\tau^{\prime}+\beta \theta_{1}\right)=1.0776$

$112-819$

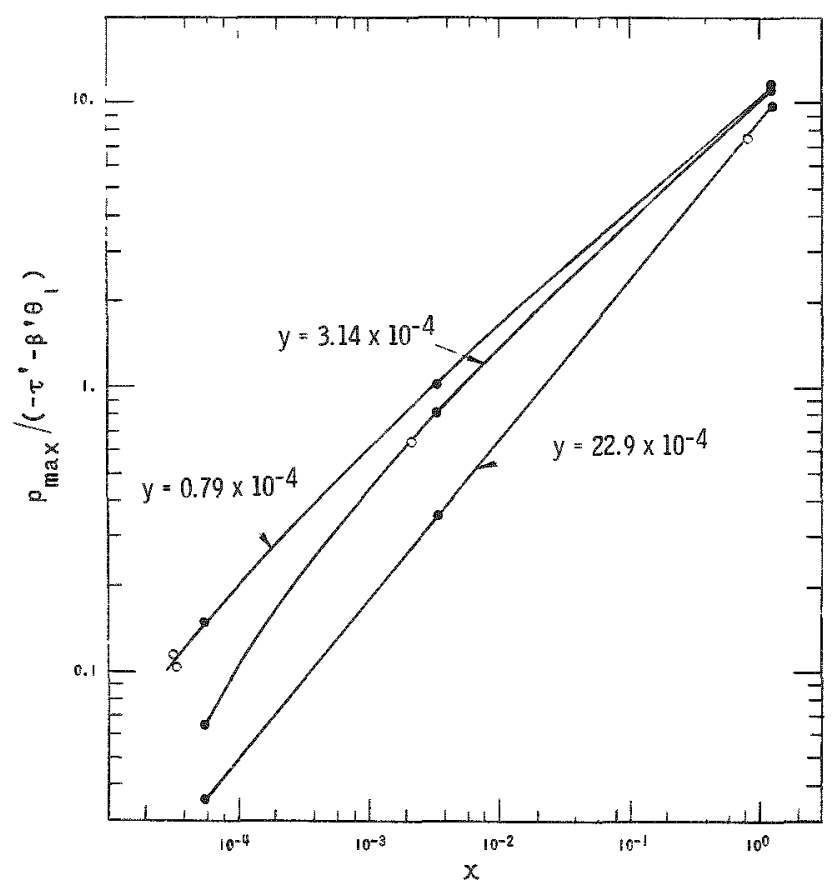

Fig. 11. Dimensionless Center Peak Pressure, $p_{\max } /\left(-\tau^{\prime}-\beta \theta_{i}\right)$, as a Function of Dimensionless Parameters $\times$ Eq. (32) and $y \mathrm{Eq} \cdot(6 \mathrm{~b}) ; \tau^{\prime} /\left(\tau^{\prime}+\beta \theta_{\mathrm{i}}\right)=1.0776$ 